\title{
Coal Technology Program Progress Report for September 1977
}

\section{OAK RIDGE NATIONAL LABORATORY}

OPERATED BY UNION CARBIDE CORPORAIION FOR THE ENERGY RESEARCH AND DFVFIOPMFNT ARMINISTRATION 


\section{DISCLAIMER}

This report was prepared as an account of work sponsored by an agency of the United States Government. Neither the United States Government nor any agency Thereof, nor any of their employees, makes any warranty, express or implied, or assumes any legal liability or responsibility for the accuracy, completeness, or usefulness of any information, apparatus, product, or process disclosed, or represents that its use would not infringe privately owned rights. Reference herein to any specific commercial product, process, or service by trade name, trademark, manufacturer, or otherwise does not necessarily constitute or imply its endorsement, recommendation, or favoring by the United States Government or any agency thereof. The views and opinions of authors expressed herein do not necessarily state or reflect those of the United States Government or any agency thereof. 


\section{DISCLAIMER}

Portions of this document may be illegible in electronic image products. Images are produced from the best available original document. 
Printed in the United States of America. Available from National Technical Information Service

U.S. Department of Commerce 5285 Port Royal Road, Springfield, Virginia 22161

Price: Printed Copy $\$ 5.25$; Microfiche $\$ 3.00$

This report was prepared as an account of work sponsored by an agency of the United States Government. Neither the United States Government nor any agency thereof, nor any of their employees, contractors, subcontractors, or their employees, makes any warranty, express or implied, nor assumes any legal liability or responsibility for any third party's use or the results of such use of any information, apparatus, product or process disclosed in this report, nor represents that its use by such third party would not-infringe privately owned rights. 
Contract No. W-7405-eng-26

COAL TECHNOLOGY PROGRAM

PROGRESS REPORT FOR SEPTEMBER 1977

Date Published - October 1977

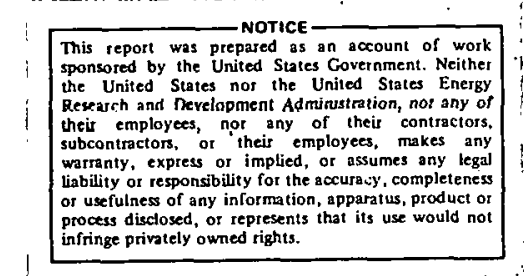

NOTICE This document contains information of a preliminary nature It is subject to revision or correction arid therefore does not represent a final report.

OAK RIDGE NATIONAL LABORATORY

Oak Ridge, Tennessee 37830 operated by UNION CARBIDE CORPORATION

for the DEPARTMENT OF F.NFRGY 
PREVIOUS REPORTS IN THIS SERIES

\author{
ORNL/TM-5044, Progress Report for August 1974 \\ ORNL/TM-5045, Progress Report for September 1974 \\ ORNL/TM-5046, Progress Report for October 1974 \\ ORNL/TM-4787, Progress Report for November 1974 \\ ORNL/TM-4796, Progress Report for December 1974 \\ ORNL/TM-4850, Progress Report for January 1975 \\ ORNL/'IM-4873, Progress Report for February 1975 \\ ORNL/TM-4892, Progress Report for March 1975 \\ ORNL/TM-4946, Progress Report for April 1975 \\ ORNL/TM-4966, Progress Report for May 1975 \\ ORNL/TM-5010, Progress Report for June 1975 \\ ORNL/TM-5037, Progress Report for July 1975 \\ ORNL/TM-5092, Progress Report for August 1.975 \\ ORNL/TM-5124, Progress Report for September 1975 \\ ORNL/TM-5186, Progress Report for October 1975 \\ ORNL/TM-5214, Progress Report for November 1975 \\ ORNL/TM-5246, Progress Report for December 1975 \\ ORNL/TM-5301, Progress Report for January 1976. \\ ORNL/TM-5321, Progress Report for February 1976 \\ ORNL/TM-5430, Progress Report for March 1976 \\ ORNL/TM-5479, Progress Report for April 1976 \\ ORNL/TM-5532, Progress Report for May 1976 \\ ORNL/TM-5595, Progress Report for June 1976 \\ ORNL/TM-5611, Progress Report for July 1976 \\ ORNL/TM-5654, Progress Report for August 1976 \\ ORNL/TM-5674, Progress Report for September 1976 \\ ORNL/TM-5717, Progress Report for October 1976 \\ ORNL/TM-5752, Progress Report for November 1976 \\ ORNL/TM-5770, Progress Report for December 1976 \\ ORNL/TM-5819, Progress Report for January 1977 \\ ORNL/TM-5858, Progress Report for February 1977 \\ ORNL/TM-5883, Progress Report for March 1977 \\ ORNL/TM-5932, Progress Report for April 1977 \\ ORNL/TM-5980, Progress Report for May 1977 \\ ORNL/TM-6003, Progress Report for June 1977 \\ ORNL/TM-6038, Progress Report for July 1977 \\ ORNL/TM-6066, Progress Report for August 1977
}




\title{
CONTENTS
}

\begin{abstract}
$\underline{\text { Page }}$
Abstract .......................... . 1

1. Summary ... . . . . . . . . . . . . . . . . 1

2. Coal Conversion Process Development . . . . . . . . . . . . . 4

2.1 Hydrocarbonization Research . . . . . . . . . . . . . 4

2.2 Experimental. Engineering Support of an
In Situ Gasification Process . . . . . . . . . . . . 11

2.3 Coal-Solvent-Hydrogen Mixing . . . . . . . . . . . . 12

2.4 Pressurized Carbonization of Coal Liquefaction Residue . . 12

2.5 References for Section 2 . . . . . . . . . . . . . . 18
\end{abstract}

3. Materials Engineering . . . . . . . . . . . . . . . . 19

3.1 Pressure Vessel and Piping Materials . . . . . . . . . . . 19

3.2 Inspection Techniques for Wear- and Process- . . Resistant Coatings .. . . . . . . . . . . . . ' 20

3.3. Fossil Energy Welding and Cladding Program . . . . . . . . 20

3.4 Fireside Corrosion . . . . . . . . . . . . . . . . . . . 21

3.5 Failure Analysis and Prevention . . . . . . . . . . . 21

4. Alkali Metal Vapor Topping Cycles . . . . . . . . . . . . 23

4.1 Gas-Fired Potassium Boiler . . . . . . . . . . . . 23

4.2 Coal-Fired Alkali Metal . . . . . . . . . . . . 24

5. Coal Equipment Test Program . . . . . . . . . . . . . 25

6. Fluidized Bed Cumbustor Télnulogy Tést Unit. . . . . . . . . . 26

7. Engineering Studies and Technical Support . . . . . . . . . . . 27

7.1 Process Modeling . . . . . . . . . . . . . . . . 27

7.2 Synthetic Fuels Process Research Digest . . . . . . . . . . 28

7.3 Survey of Industrial Coal Conversion Capabilities . . . . . . 28

7.4 Engineering Assistance . . . . . . . . . . . . . . . 30

7.5 Flash Hydropyrolysis . . . . . . . . . . . . . . 31

7.6 Hot Gas Purification Processes . . . . . . . . . . . . . . 31

7.7 Large Oxygen Plant Study . . . . . . . . . . . . . . . 33

7.8 State-of-the-Art Review of Heat Recovery Processes . . . . . 35

7.9 Assessment of a Moving Bed System for Cleanup 
7.10 Technica1/Economic Assessment of Hydrogen

Production by the Steam/Molten Iron Process . . . . . . . . 36

7.11 A Study of Effluent Control Technologies for Hydrocarbon and Carbon Monoxide Emissions from Coal Conversion Plants . 37

8. Process and Program Analysis . . . . . . . . . . . . . . 41

8.1 Low Btu Coal Gasification . . . . . . . . . . . . 41

8.2 Direct Combustion .................. . . 43

8.3 Advanced Power Conversion Systems . . . . . . . . . . 43

8.4 Liquefaction . . . . . . . . . . . . . . . . 43

8.5 High Btu Gas . . . . . . . . . . . . . . . . . . . 44

8.6 In Situ Coal Gasification . . . . . . . . . . . . 45

8.7 Coal Beneficiation................... 45

8.8 Gas Cleanup Studies . . . . . . . . . . . . . . 46

8.9 The Potential of the Synthesis of Chemicals trom
Coal as a DOE Research Program . . . . . . . . . 46

9. Fossil Energy Environmental Project . . . . . . . . . . . . 47

9.1 Landfill Storage of Coal Conversion

9.2 Landfill Storage of Coal Conversion
Solid Wastes/Experimental Phase . . . . . . . . . . . 47

9.3 Environmental Monitoring Handbook . . . . . . . . . . 47

9.4 Programmatic Assessment/Pipeline Gas . . . . . . . . . . 48

9.5 Programmatic Assessment/Fuel Gas . . . . . . . . . . . 48

9.6 Technical Assistance. . . . . . . . . . . . . . . 48 


\section{ABSTRACT}

This report - the thirty-eighth of a series - is a compendium of monthly progress reports for the ORNL research and development programs that are in support of the increased utilization of coal as a source of clean energy. The projects reported this month include those for coal conversion process development, materials engineering, alkali metal vapor topping cycles, a coal equipment test facility, a fluidized bed combustor technology test unit, engineering and support studies, process and program assistance, and environmental assessment studies.

\section{SUMMARY}

J. P. Nichols

Highlights of our progress in September are as follows:

- A successful hydrocarbonization experiment at 300 psi of hydrogen and $\sim 1050^{\circ} \mathrm{F}$ was completed with Illinois No. 6 coal that had been chemically pretreated with aqueous $\mathrm{CaO}$ and $\mathrm{NaOH}$. Difficulties with agglomeration were eliminated. The next run will employ coal treated with $\mathrm{Na}_{2} \mathrm{CO}_{3}$ which may enhance the production of oil as well.

- Experimental engineering support of in situ gasification was delayed by a malfunction of the heater controller and a resultant heater failure. Repairs are nearly completed.

- In our coal-solvent-hydrogen mixing work, final fluid dynamics data are presented. Analysis and correlation of these data will be presented in a final summary report which is in preparation.

- In pressurized carbonization, one successful experiment at $\sim 1.100^{\circ} \mathrm{F}$ and $415 \mathrm{psi}$ of methane was completed with vacuum distillation residue from the H-Coal process. This difficult feed material produced a small amount of very heavy tar and caused significant agglomeration in the equipment. 
- In our thick section pressure vessel work, procedures are being developed with the DATA TRAK heat treating facility to allow preparation of relatively large heat treated samples; $21 / 4 \mathrm{Cr}-1$ Mo steel samples are being heat treated.

- We are currently planning work on CoCrAlY coatings for FY 1978. After some initial difficulties, we have apparently located a supplier for the CoCrAlY material. We are completing some ultrasonic work on ceramic and cermet coatings which will carry over to the metallic coatings.

- Cladding development work with type 320 stainless steels has focused on the effect of weld wire diameter on depnsit. integrity. The use of Inconel 82 to form intermediate layers in cladding is now being studied.

- Testing of heat exchanger tube materials in the Fluidyne fluidized bed test unit continues.

- Fallure analysis and prevention work has continued with emphasis on solvent refined coal plants. Examination of erosion-corrosion test coupons exposed to a simulated gasifier environment at the Solar Corporatton was starced.

- In our Gas-Fired Potassium Boiler Project, the potassium system installation was completed, the fill and drain tank was filled with potassium, and the checkout of the instruments and controls was nearly completed. Initial filling of the boiler with potassium is planned for about October 12, and shakedown tests will begin after that.

- The Coal-Fired Alkali Metal Power System Design Study was completed in September and a draft report describing the design was issued. Cesium was selected as the working fluid for the topping cycle. For the reference design, the furnace operated at atmospheric pressure and the cycle conditions for the power conversion systems were $1500^{\circ} \mathrm{F}$ $(1089 \mathrm{~K})$ to $900^{\circ} \mathrm{F}(756 \mathrm{~K})$ for the topping cycle and $2400 \mathrm{psi}(16.5 \mathrm{MPa}) /$ $1000^{\circ} \mathrm{F}(811 \mathrm{~K}) / 1000^{\circ} \mathrm{F}(811 \mathrm{~K})$ to $11 / 2 \mathrm{in}$. $\mathrm{Hg}(5079 \mathrm{~Pa})$ for the steam system.

- TRW Energy Systems completed their subcontract activity to develop a comprehensive plan for the Coal Equipment Test Program (CETP).

- ORNL was requested by DOE to develop a program for testing coal feeders currently under development. A meeting was held at ORNL with Jet Propulsion Laboratory (JPL) personnel who have been coordinating this activity for MFPM. Agreement was reached that JPL will prepare the criteria for the feeder test program. Upon receipt of these criteria at the end of October, ORNL will proceed with the selection of a site and prepare a preliminary plan for a feeder test program. 
- Preparation of the performance specifications for the Fluidized Bed Combustor Technology Test Unit continued. The specification for the conceptual design was completed. It was reviewed internally and has been revised. The specification for the detailed design phase was completed and is now being reviewed.

- In our engineering studies and evaluations project, we continued work on process modeling, the preparation of a Synthetic Fuels Research Digest, a survey of industrial coal conversion equipment capabilities, and studies of flash hydropyrolysis, hot gas purification processes, processes for heat recovery, and hydrogen production by the steam/molten iron process.

- In our process and program analysis studies, we continued studies of low-Btu gasification, direct combustion, advanced power conversion systems, liquefaction, high-Btu gasification, in situ gasification, and coal beneficiation. These initial scoping studies are due to be complete with the issuance of reports by October 1 .

- Fossil Energy Environmental Project members participated in a site visit to the CONOCO demo plant location and in a two-day work session at Westinghouse Environmental Systems Department (WESD), Pittsburgh, to assist the environmental subcontractor in developing a detailed monitoring program. 


\section{COAL CONVERSION PROCESS DEVELOPMENT}

H. D. Cochran, Jr.

Coal conversion process development activities are carried out in the Chemical Technology Division. This section discusses hydrocarbonization studies in a 20-atm bench-scale facility, engineering support studies for in situ gasification, studies for identifying and characterizing alternate reactor concepts for coal hydroliquefaction, and studies of residue carbonization in a pressurized carbonizer.

\subsection{Hydrocarbonization Research}

R. L. Andrews, J. Beams, C. H. Brown, Jr., L. S. Dickerson, J. B. Gibson, G. E. Oswald, J. C. Rose, T. C. Scott, P. R. Westmoreland, and E. L. Youngblood

\subsubsection{Experimental Development}

Work Accomplished. Analysis of data from recirculating fluidized bed experiments continued during September. These data include the effect of gas and solid properties but do not include any effects of changes in reactor configuration or geometry. Char from experiment HC-12 (solid properties were described earlier 1,2 ) was fluidized by helium or nitrogen in a 4-in.-diam model of a recirculating fluidized bed reactor as described in Table 2.1.

Table 2.1. Dimensions and configuration of recirculating fluidized bed reactor model

Reactor:

Draft tube:

Draft tube gas supply nozzle:

Distributor plate:
$10.16 \mathrm{~cm}$ ( 1 in.) inside diameter

$133.4 \mathrm{~cm}$ (52-1/2 in.) long

$2.54 \mathrm{~cm}$ (1 in.) inside diameter

$0.318 \mathrm{~cm}$ ( $1 / 8$ in.) wall thickness

$63.5 \mathrm{~cm}$ (25 in.) long

$2.54 \mathrm{~cm}$ (1 in.) above distributor plate

$0.0876 \mathrm{~cm}^{2}$ cross-sectional area (3/16-in. O.D., 0.028-in. wall tubing)

Aligned with draft tube axis

Flush with distributor plate

42 holes spaced under the downcomer on a $1.19 \mathrm{~cm}$ (15/32 in.) triangular pitch

Holes 397-jm-diam (1/64-in.) 
Analysis of solid circulation rate data using dimensionless gas velocity (the ratio of gas velocity to minimum fluidization velocity, $\mathrm{u} / \mathrm{u}_{\mathrm{mf}}$ ) was reported earlier. ${ }^{3}$ (Experimental data are summarized in Table 2.2.) By plotting $u / u_{m f}$ for the draft tube gas supply against $\mathrm{u} / \mathrm{u}_{\mathrm{mf}}$ for the downcomer gas supply (the two manipulated variables), a matrix of test conditions was graphed. Such a graph shows reasonable agreement of pressure drop and of solid circulation rate between the two different gases.

Figure 2.1 shows the solid circulation rate data presented in this fashion. The effect of $u / u_{\mathrm{mf}}$ to the downcomer is strongly pronounced. Increasing $u / u_{m f}$ to the draft tube has a smaller effect on circulation rate. As shown in Fig. 2.2 (a plot of circulation rate data as a function of gas supply rate to the downcomer), a twenty-fold varlation of draft tube gas supply results in only a slight deviation of the general trend.

Char circulation rate appears to be limited in Fig. 2.2. Restriction of solids flow in some cross-section of solids flow-path--downcomer cross-sectional area, draft tube-distributor plate spacing, or draft tube area--probably causes the limitation. Adjustment of these geometric factors could increase the solid circulation rates achievable.

Work Forecast. The 4-in.-diam model will be lengthened so that effects of a longer draft tube may be determined.

\subsubsection{Bench-Scale Hydrocarbonization}

Work Accomplished. During September run $\mathrm{HC}-23$ was made using Illinois No. 6 coal that had been pretreated with an aqueous mixture of lime and sodium hydroxide.

A total of 23 batches of Illinois No. 6 coal were treated in preparation for run HC-23. Each batch was prepared by mixing $1.81 \mathrm{~kg}$ of coal $(-50+170$ mesh) with $0.24 \mathrm{~kg}$ of $\mathrm{CaO}, 1.27 \mathrm{~kg}$ of $\mathrm{NaOH}$, and $7.28 \mathrm{~kg}$ of water. The mixture was heated in a rocking autoclave to $200-275^{\circ} \mathrm{C}$ for approximately one hour. After removal from the autoclave the coal was washed with water, filtered, and dried in a vacuum drier. The treated coal had a Free-Swelling Index of less than 1 with only a slight tendency to agglomerate. The first treated coal (11.8 kg) that was prepared ignited spontaneously when exposed to air after removal from the drier. This material was discarded. The remainder of the treated coal was removed from the drier directly into an argon atmosphere to prevent oxidation.

The treated coal was passed through a Sweco Separator to remove oversize ( +38 mesh) particles. After the oversize particles were removed, the remaining coal contained about $31 \%$ fines ( -140 mesh). Apparently the caustic treatment had caused considerable comminution of the coal particles. A small portion of the material was tested in the feeder 
Table 2.2. Data for evaluation of recirculating fluidized bed flow rate correlction.

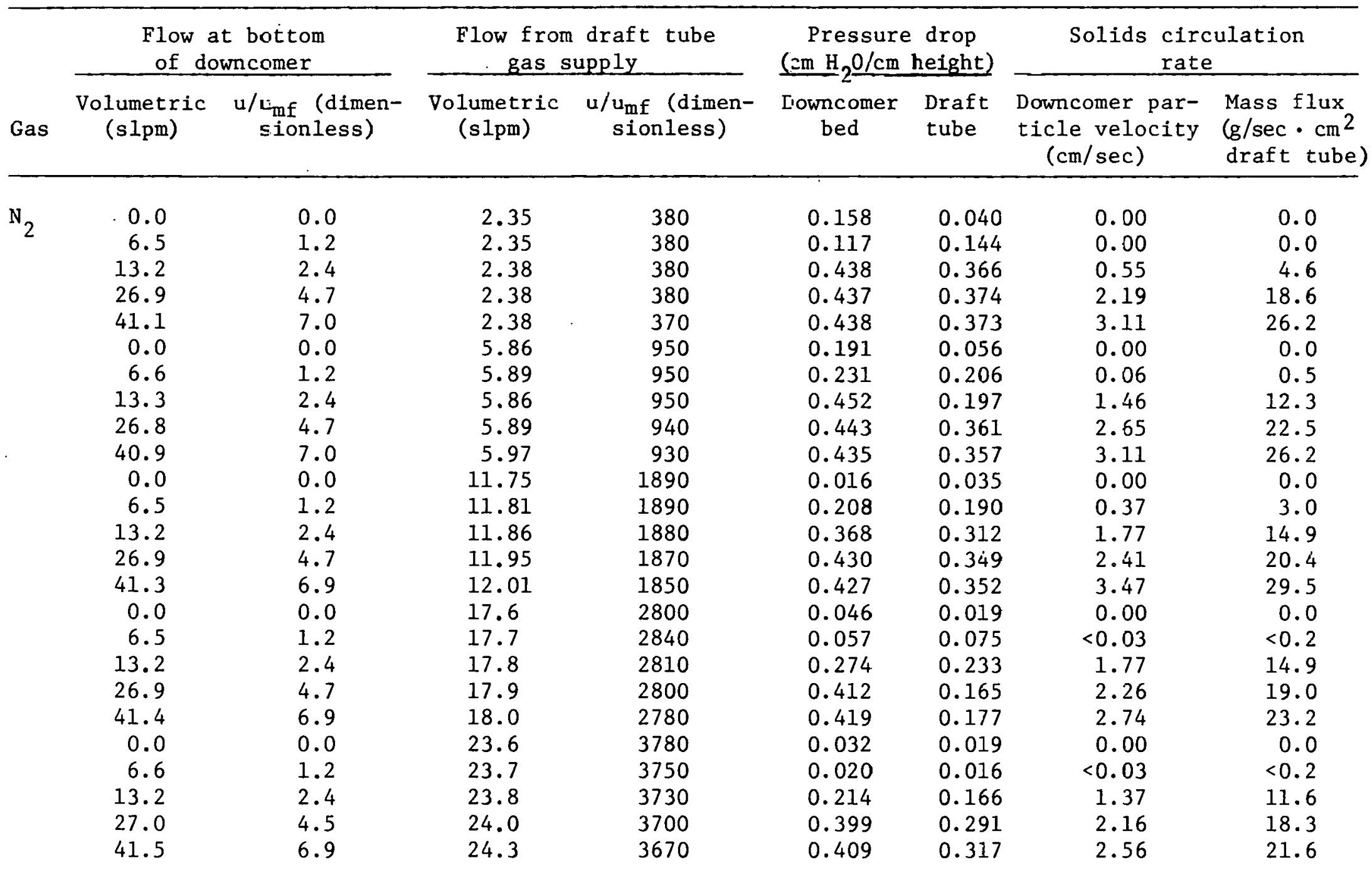


Table 2.2. (Continued)

\begin{tabular}{|c|c|c|c|c|c|c|c|c|}
\hline \multirow[b]{2}{*}{ Gas } & \multicolumn{2}{|c|}{$\begin{array}{l}\text { Flow at bottom } \\
\text { of downcomer }\end{array}$} & \multicolumn{2}{|c|}{$\begin{array}{c}\text { Flow from draft tube } \\
\text { gas supply }\end{array}$} & \multicolumn{2}{|c|}{$\begin{array}{l}\text { Pressure drop } \\
\text { (cm } \mathrm{H}_{2} \mathrm{O} / \mathrm{cm} \text { height) }\end{array}$} & \multicolumn{2}{|c|}{$\begin{array}{l}\text { Solids circulation } \\
\text { rate } \\
\end{array}$} \\
\hline & $\begin{array}{l}\text { Volumetric } \\
\text { (slpm) }\end{array}$ & $\begin{array}{l}\mathrm{u} / \mathrm{u}_{\mathrm{mf}} \text { (dimen- } \\
\text { sionless) }\end{array}$ & $\begin{array}{l}\text { Volumetric } \\
\quad(\mathrm{s} 1 \mathrm{pm})\end{array}$ & $\begin{array}{l}\mathrm{u} / \mathrm{u}_{\mathrm{mf}} \text { (dimen- } \\
\text { sionless) }\end{array}$ & $\begin{array}{l}\text { Downcomer } \\
\text { bed }\end{array}$ & $\begin{array}{l}\text { Draft } \\
\text { tube }\end{array}$ & $\begin{array}{l}\text { Downcomer par- } \\
\text { ticle velocity } \\
\quad(\mathrm{cm} / \mathrm{sec})\end{array}$ & $\begin{array}{l}\text { Mass flux } \\
\left(\mathrm{g} / \mathrm{sec} \cdot \mathrm{cm}^{2}\right. \\
\text { draft tube) }\end{array}$ \\
\hline $\mathrm{He}$ & $\begin{array}{l}35 . \\
70 . \\
80 . \\
17 . \\
35 . \\
70 . \\
17 . \\
35 . \\
70 . \\
17 . \\
15 . \\
70 . \\
17 . \\
35 . \\
70 .\end{array}$ & $\begin{array}{r}5.1 \\
10.3 \\
11.8 \\
2.6 \\
5.1 \\
10.3 \\
2.6 \\
5.1 \\
10.3 \\
2.6 \\
5.1 \\
10.3 \\
2.6 \\
5.1 \\
10.3\end{array}$ & $\begin{array}{r}6.3 \\
6.3 \\
6.3 \\
15.8 \\
15.8 \\
15.8 \\
31.6 \\
31.6 \\
31.6 \\
47.5 \\
47.5 \\
47.5 \\
63.3 \\
63.3 \\
63.3\end{array}$ & $\begin{array}{r}800 \\
800 \\
800 \\
2020 \\
2020 \\
2020 \\
4030 \\
4030 \\
4030 \\
6040 \\
6040 \\
6040 \\
8060 \\
8060 \\
8060\end{array}$ & $\begin{array}{l}0.430 \\
0.411 \\
0.389 \\
0.448 \\
0.418 \\
0.408 \\
0.461 \\
0.407 \\
0.399 \\
0.380 \\
0.417 \\
0.399 \\
0.376 \\
0.428 \\
0.415\end{array}$ & $\begin{array}{l}0.369 \\
0.382 \\
0.408 \\
0.373 \\
0.374 \\
0.373 \\
0.328 \\
0.345 \\
0.358 \\
0.280 \\
0.310 \\
0.291 \\
0.240 \\
0.299 \\
0.305\end{array}$ & $\begin{array}{l}2.26 \\
2.35 \\
3.35 \\
2.04 \\
4.54 \\
3.47 \\
2.04 \\
4.63 \\
4.39 \\
1.68 \\
2.93 \\
3.69 \\
1.22 \\
2.74 \\
2.74\end{array}$ & $\begin{array}{r}17.2 \\
17.9 \\
25.5 \\
15.5 \\
34.6 \\
26.4 \\
15.5 \\
35.3 \\
33.4 \\
12.8 \\
22.3 \\
28.1 \\
9.3 \\
20.9 \\
20.9\end{array}$ \\
\hline
\end{tabular}


ORNL DWG 77-1836

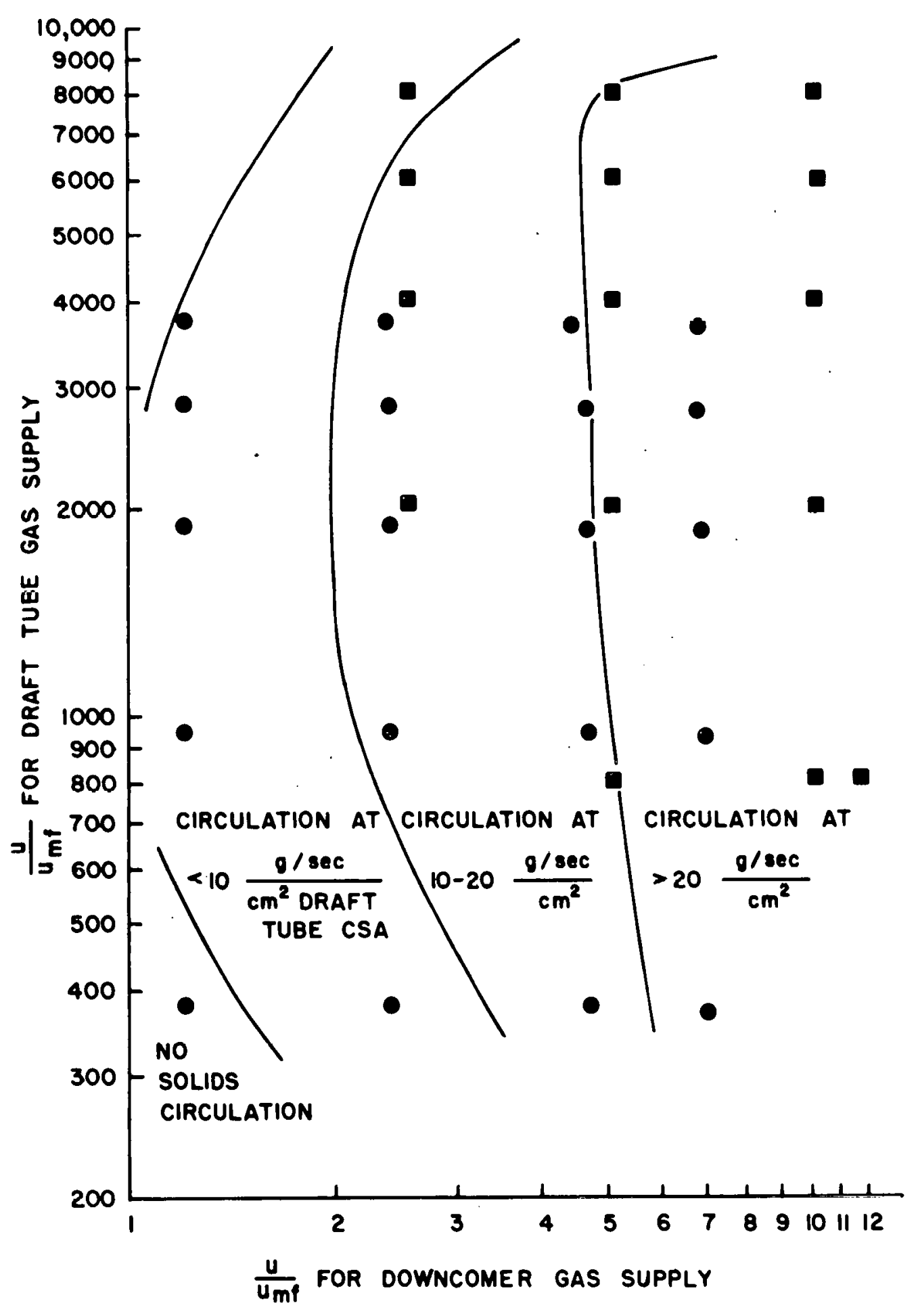

Fig. 2.1. Solids circulation in a 4-in.-diam recirculating fluidized bed -- interdependence of draft tube and downcomer gas supply rates (Nitrogen, Helium). 
ORNL DWG 77-1834

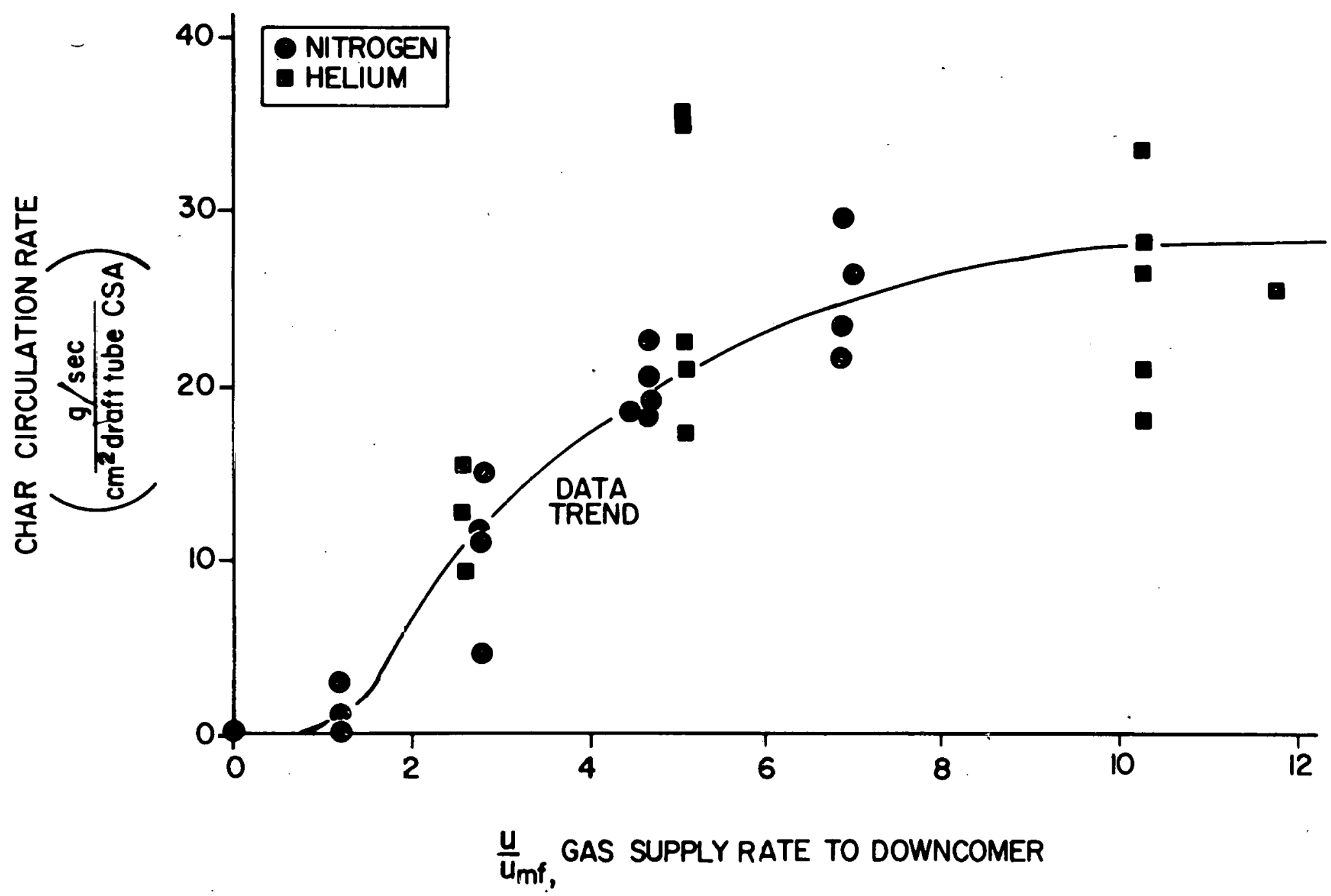

Fig. 2.2. Solids circulation in a 4-in.-diam recirculating fluidized bed -- dependence on downcomer gas supply rate. 
to the Bench-Scale Hydrocarbonization System and was found to have poor flow characteristics. The fines content of the coal was then reduced to $25 \%$ by one pass through the Sonic-Sifters. This material had acceptable flow characteristics and was used as the feed material for the run.

The operating conditions for run HC-23 were essentially the same as those used in the previous run ( $\mathrm{HC}-22$ ) with the exception that treated coal was used as feed material. The draft tube was used to provide recirculation of the reactor bed. Prior to the run, 9.3 lbs of char was fed to the reactor for the starting bed. The reactor temperature was held at $1060^{\circ} \mathrm{F}$ throughout the run. The average $\mathrm{H}_{2}$ flow rate to the coal transport line was $4.4 \mathrm{scfm}$ and the $\mathrm{H}_{2}$ rates to the downcomer and draft tube were $14.1 \mathrm{scfm}$ and $1.5 \mathrm{scfm}$ respectively. The coal feed stream entering the reactor was preheated to $375^{\circ} \mathrm{F}$.

Operation of the system was generally good with the exception that the coal feed rate was lower than planned, possibly due to the large amount of fines present in the feed. Fluidization of the bed appeared to be good throughout the run as indicated by uniform temperatures in the bed and a constant pressure differential across the bed. The temperature of the material circulating through the draft tube was only about 15 to $20^{\circ} \mathrm{F}$ lower $\left(1040^{\circ} \mathrm{F}\right)$ than the temperature of the downcomer region indicating that there was good circulation of char through the draft tube. There were no indications of caking of the bed with the treated coal as had been observed in previous runs with untreated Illinois No. 6 coal. From the start of the run the coal feed rate was less than planned. In an attempt to improve the feed rate the feed hopper was pressurized slightly. After 4 hours of operation the system pressure was reduced from $300 \mathrm{psig}$ to $280 \mathrm{psig}$ to avoid the possibility of blowing a rupture disc. The run continued for 13.3 hours until all the coal ( 63 lbs) was fed. The average feed rate for the run was $4.7 \mathrm{lb} / \mathrm{hr}$.

Upon completion of the run the system was drained and samples were taken for analysis. Analytical results and material balances will be presented in the next monthly report. Visual examination of the o1l from the scrubber indicates the possibility of a higher than normal carry-over of char from the reactor to the scrubbers due to the large quantity of fines in the feed material.

A composite oil sample from run $\mathrm{HC}-21$, which was made using Wyodak coal in a uniformly fluidized bed at $1035^{\circ} \mathrm{F}$, was separated into 10 fractions using gel partition. (The fractionation was done without charge to the program.) The results presented in Table 2.3 will be useful in evaluating the products produced in the Bench-Scale Hydrocarbonization System and in determining the effect of operating var1ables on the product produced. Also, a sample of the heavy oil from run $\mathrm{HC}-12$ has been analyzed for Benzo (a) pyrene (BaP) by the Analytical Chemistry Division. The $\mathrm{BaP}$ content of the oil was determined to be $1.8 \mathrm{mg} / \mathrm{g}$. 
Work Forecast. The next run will be made using Illinois No. 6 coal that has been treated with sodium carbonate. The treatment is expected to eliminate the caking characteristics of the coal and to improve oil yield from hydrocarbonization. Work is already underway to prepare treated coal for the run. It is planned to operate the Bench-Scale Hydrocarbonization System at a reduced manpower level during the next fiscal year. Most of the operating crew will be assigned to other projects except during the time that runs are being made. The next hydrocarbonization run is scheduled to be made around the first of December.

Table 2.3. Fractionation of oil. from Run HC-21 by the Gel Partition Method

\begin{tabular}{lc}
\multicolumn{1}{c}{ Fraction } & Wt $\%$ \\
\hline Water and other volatiles & 4.0 \\
Hexane Insolubles & 33.2 \\
Particulate Matter $(>5 \mathrm{u})$ & 0.02 \\
Hydrophilic (polar) & 17.8 \\
Polymeric ( $5000 \mathrm{mw})$ & 8.5 \\
H-Bonding & none detected \\
Aliphatic & 8.0 \\
Monoaromatic & 12.3 \\
Di- and Triaromatic & 9.8 \\
Polyaromatic & 10.2 \\
\hline
\end{tabular}

2.2 Experimental Engineering Support of an In Situ Gasification Process

J. B. Gibson, P. R. Westmoreland, and L. S. Dickerson

As described in previous reports, samples of Eastern bituminous coal have been obtained from the Morgantown Energy Research Center (MERC) for use in our large-block pyrolysis studies. The coal is typical of the material which will be burned by MERC in their fleld test near Pricetown, West Virginia. 
During September one run was attempted. The temperature controller malfunctioned during this run and a heater on the reactor was burned out. The heater has been repaired, and the controller is in the instrument shop. The possibility of replacing the current controller with a more modern one is now being investigated.

An available process gas chromatograph is being installed in the in situ system. This chromatograph will provide a rapid analysis of the gases evolved during gasification and should greatly reduce turnaround time and analytical costs for the experiment.

Analytical results were recelved for runs BP2-32, BP2-33, and BP2-34 near the end of the month. These will be analyzed and reported in the next progress report.

\subsection{Coal-Solvent-Hydrogen Mixing}

J. M. Begovich and R. C. Lovelace

Pulse test measurements in the 1-in.-ID x 5-ft-long Kenics contactor using cocurrent upflow of air and liquid have now been completely analyzed. A total of 118 runs were made--58 using air and water and 60 using air and a $35 \mathrm{wt} \% \mathrm{coal} /$ water slurry. The results of the analysis are presented in Tables 2.4 and 2.5 for the air/water and air/coal/water systems, respectively.

A group of graduate students of the Massachusetts Institute of Technology's School of Chemical Engineering Practice (MIT-SCEP) obtained mixing, holdup, and pressure drop data on several systems during September. 4 Systems studied included the following: (I) a 0.5-in.-ID $x$ 8-ft-long open column using first water then coal/aqueous glycerol slurries and air in cocurrent upflow, and (2) a 1-in.-ID x 5-ft-long column packed with 1/8-in. $x$ 1/8-in. cylindrical packing using first water then a 35 wt \% coal/water slurry and air in cocurrent upflow. Preliminary observations indicate that the liquid viscosity has little effect upon the liquid saturation in an open column, while cylindrical pellets in a packed column increase both the liquid saturation and the energy dissipation per unit volume as compared to spherical packing.

\subsection{Pressurized Carbonization of Coal Liquefaction Renidue}

R. E. Barker and S. M. Gibson

This project is intended to determine experimentally the degree of coking and liquid degradation to be expected when coal liquefaction residues are carbonized at pressures greater than atmospheric. The experimental apparatus and earlier experiments have been described previously. 2 
Table 2.4. Results of RTD and pressure drop experiments in a Kenics contactor with cocurrent upflow of air and water

Column Diameter $=2.54 \mathrm{~cm}$

Column Length $=160 \mathrm{~cm}$

\begin{tabular}{|c|c|c|c|c|c|c|c|c|}
\hline $\begin{array}{c}\text { Run } \\
\text { number }\end{array}$ & $\begin{array}{l}\text { Gas } \\
\text { flow } \\
\mathrm{cm}^{3} / \mathrm{sec}\end{array}$ & $\begin{array}{l}\text { Liquid } \\
\text { flow } \\
\mathrm{cm}^{3} / \mathrm{sec}\end{array}$ & $\begin{array}{l}\text { Liquid holdup, } \varepsilon_{L} \\
\text { (fraction of } \\
\text { total bed volume) }\end{array}$ & $\begin{array}{l}\text { Liquid saturation, } \\
R_{L} \text { (fraction of } \\
\text { void volume) }\end{array}$ & $\begin{array}{l}\text { Peclet } \\
\text { number } \\
\mathrm{Pe}\end{array}$ & $\begin{array}{l}\text { Dispersion } \\
\text { coefficient, } \mathrm{D}_{\mathrm{L}} \\
\mathrm{cm}^{2} / \mathrm{sec}\end{array}$ & $\begin{array}{l}\text { Pressure } \\
\text { drop } \\
\Delta \mathrm{P} \\
\text { in. } \mathrm{H}_{2} \mathrm{O}\end{array}$ & $\begin{array}{l}\text { Energy dissipation } \\
\text { per unit volume } \\
\frac{\mathrm{v}_{\mathrm{L}}^{\Delta \mathrm{P}_{\mathrm{f}}}}{\mathrm{L}^{-3}} \\
\mathrm{ft} \cdot \mathrm{lb}_{\mathrm{f}} \cdot \mathrm{ft}^{-1} \cdot \mathrm{sec}^{-1}\end{array}$ \\
\hline 266 & 0 & 1.46 & 0.849 & 1.010 & 279.4 & 0.2 & 0 & 0 \\
\hline 267 & 0 & 2.36 & 0.841 & 1.001 & 420.8 & 0.2 & 0 & 0 \\
\hline 268 & 0 & 3.03 & 0.840 & 1.000 & 409.7 & 0.3 & 0 & 0 \\
\hline 269 & 0 & 3.75 & 0.820 & 0.976 & 954.1 & 0.2 & 0 & 0 \\
\hline 276 & 0 & 1.63 & 1.028 & 1.224 & 242.3 & 0.2 & 0 & 0 \\
\hline 277 & 0 & 2.38 & 1.041 & 1.239 & 114.4 & 0.6 & 0 & 0 \\
\hline 278 & 0 & 3.17 & 0.873 & 1.039 & 157.3 & 0.7 & 0 & 0 \\
\hline 279 & 0 & 3.83 & 0.900 & 1.071 & 85.2 & 1.6 & 0 & 0 \\
\hline 280 & 0 & 5.83 & 0.887 & 1.056 & 187.0 & 1.1 & 0 & 0 \\
\hline 281 & 0 & 9.67 & 0.860 & 1.023 & 175.9 & 2.0 & 0.1 & 0 \\
\hline 283 & 0 & 17.75 & 0.865 & 1.029 & 303.5 & 2.1 & 0.6 & 0.1 \\
\hline 284 & 0 & 21.25 & 0.862 & 1.026 & 359.4 & 2.2 & 0.8 & 0.1 \\
\hline 285 & 116.5 & 21.50 & 0.459 & 0.547 & 107.9 & 13.7 & 0 & 8.6 \\
\hline 286 & 116.5 & 21.75 & 0.472 & 0.561 & 109.9 & 13.3 & 0 & 8.1 \\
\hline 287 & 116.5 & 17.50 & 0.461 & 0.549 & 66.3 & 18.1 & 0 & 6.9 \\
\hline 288 & 116.5 & 13.67 & 0.438 & 0.522 & 83.5 & 11.8 & 0 & 6.0 \\
\hline 289 & 120.3 & 9.56 & 0.409 & 0.487 & 59.1 & 12.5 & 0 & 4.8 \\
\hline 290 & 121.3 & 5.83 & 0.394 & 0.469 & 45.1 & 10.4 & 0 & 3.2 \\
\hline 291 & 214.8 & 21.00 & 0.389 & 0.464 & 90.7 & 18.8 & 0 & 11.7 \\
\hline 292 & 212.2 & 21.25 & 0.398 & 0.474 & 104.8 & 16.1 & 0 & 11.3 \\
\hline 293 & 214.8 & 17.67 & 0.390 & 0.464 & 111.1 & 12.9 & 0 & 9.8 \\
\hline 294 & 214.8 & 17.33 & 0.389 & 0.463 & 141.2 & 10.0 & $\therefore 0$ & 9.7 \\
\hline 295 & 217.6 & 13.58 & 0.354 & 0.421 & 99.3 & 12.2 & 0 & 9.0 \\
\hline 296 & 217.6 & 13.67 & 0.377 & 0.449 & 82.5 & 13.9. & 0 & 8.1 \\
\hline
\end{tabular}


Table 2.4. (Continued)

\begin{tabular}{|c|c|c|c|c|c|c|c|c|c|}
\hline $\begin{array}{l}\text { Run } \\
\text { number }\end{array}$ & $\begin{array}{l}\text { Gas } \\
\text { flow } \\
\mathrm{cm}^{3} / \mathrm{sec}\end{array}$ & $\begin{array}{l}\text { Liquid } \\
\mathrm{f} \text { 年w } \\
\mathrm{cm} / \mathrm{sec}\end{array}$ & $\begin{array}{l}\text { Liquid holdup, } \varepsilon_{L} \\
\quad \text { (fraction of } \\
\text { total bed volume) }\end{array}$ & $\begin{array}{l}\text { Liquid saturation, } \\
\mathrm{R}_{L_{L}} \text { (Eraction of } \\
\text { void volume) } \\
\end{array}$ & $\begin{array}{l}\text { Peclet } \\
\text { number } \\
\text { Pe }\end{array}$ & $\begin{array}{l}\text { Dispezsion } \\
\text { coefficient, } D_{L} \\
\mathrm{~cm}^{2} / \mathrm{s} \equiv \mathrm{c}\end{array}$ & $\begin{array}{l}\text { Pressure } \\
\text { Arop } \\
\quad \Delta \mathrm{P} \\
\text { in. } \mathrm{H}_{2} \mathrm{O}\end{array}$ & $\begin{array}{r}\text { Energy dissip } \\
\text { per unit vol } \\
\frac{\mathrm{v}_{\mathrm{L}} \Delta \mathrm{P}_{\mathrm{f}}}{\mathrm{L}^{-3}} \\
\mathrm{ft} \cdot 1 \mathrm{~b}_{\mathrm{f}} \cdot \mathrm{ft}^{-3}\end{array}$ & $\begin{array}{l}\text { ation } \\
\text { ume } \\
\sec ^{-1} \\
\end{array}$ \\
\hline 297 & 218.8 & 9.78 & 0.344 & 0.410 & 45.7 & 19.6 & 0 & 6.8 & \\
\hline 298 & 218.8 & 9.56 & 0.328 & 0.390 & 65.0 & 14.2 & 0 & 7.2 & \\
\hline 299 & 221.2 & 5.83 & 0.309 & 0.368 & 78.2 & 7.6 & 0 & 4.8 & . \\
\hline 300 & 221.2 & 5.83 & 0.323 & 0.384 & 71.8 & 7.9 & 0 & 4.5 & \\
\hline 301 & 300.0 & 21.00 & 0.357 & 0.425 & 109.3 & 17.0 & 0 & 13.6 & \\
\hline 302 & 300.0 & 21.00 & 0.357 & 0.425 & 91.3 & 20.4 & 0 & 13.6 & \\
\hline 303 & 305.3 & 17.50 & 0.339 & 0.403 & 93.5 & 17.5 & 0 & 12.4 & \\
\hline 304 & 305.3 & 17.33 & 0.329 & 0.391 & 97.9 & 17.0 & 0 & 12.9 & \\
\hline 305 & 312.3 & 13.67 & 0.322 & 0.384 & 71.6 & 18.7 & 0 & 10.6 & \\
\hline 306 & 312.3 & 13.75 & 0.329 & 0.392 & 82.7 & 16.0 & 0 & 10.3 & \\
\hline 307 & 318.5 & 9.44 & 0.310 & 0.369 & 51.2 & 18.8 & 0 & 7.8 & \\
\hline 308 & 318.5 & 9.44 & 0.302 & 0.360 & 82.3 & 12.0 & 0 & 8.1 & \\
\hline 309 & 323.7 & 5.67 & 0.292 & 0.347 & 44.0 & 13.9 & 0 & 5.1 & \\
\hline 310 & 323.7 & 5.75 & 0.293 & 0.349 & 50.5 & 12.3 & 0 & 5.2 & \\
\hline 311 & 401.2 & 21.25 & 0.293 & $0.348^{\circ}$ & 158.7 & 14.5 & 0 & 19.1 & \\
\hline 312 & 401.2 & 21.38 & 0.304 & 0.362 & 161.7 & 13.7 & 0 & 18.1 & \\
\hline 313 & 412.7 & 17.75 & 0.297 & 0.353 & 811 & 23.3 & 0 & 15.6 & \\
\hline 314 & 412.7 & 17.75 & 0.289 & 0.344 & 75.4 & $25 \cdot 7$ & 0 & 16.3 & \\
\hline 315 & 421.0 & 13.58 & 0.291 & 0.345 & 77.1 . & 19.1 & 0 & 12.3 & \\
\hline 316 & 421.0 & 13.83 & 0.295 & 0.352 & 69.5 & 21.3 & 0 & 12.3 & \\
\hline 317 & 437.6 & 9.67 & 0.277 & 0.329 & 59.3 & 18,6 & 0 & 9.4 & \\
\hline 318 & 437.6 & 9.72 & 0.268 & 0.319 & 59.8 & 19.1 & 0 & 10.0 & \\
\hline 319 & 447.6 & 5.83 & 0.248 & 0.295 & 49.2 & 15.1 & 0 & 6.7 & \\
\hline 320 & 447.6 & 5.83 & 0.238 & 0.284 & 627 & 12.3 & 0 & 7.1 & \\
\hline 321 & 503.5 & 21.25 & 0.279 & 0.332 & 1164 & 20.7 & 0 & 20.5 & \\
\hline 322 & 503.5 & 21.00 & 0.280 & 0.333 & 1928 & 12.3 & 0 & 20.2 & \\
\hline 323 & 514.3 & 17.17 & 0.257 & 0.306 & 185.3 & 11.4 & 0 & 1.8 .7 & \\
\hline 324 & 514.3 & 17.33 & 0.260 & 0.309 & 157.0 & 13.4 & 0 & 18.6 & \\
\hline 325 & 532.0 & 13.83 & 0.265 & 0.315 & 59.3 & 27.8 & 0 & 14.4 & \\
\hline 326 & 532.0 & 13.75 & 0.246 & 0.293 & 100.9 & 17.5 & 0 & 16.0 & \\
\hline 327 & 348.3 & 9.72 & 0.243 & 0.289 & 99.1 & 12.7 & 0 & 11.5 & \\
\hline 328 & 548.3 & 9.78 & 0.245 & 0.291 & 62.7 & 20.1 & 0 & 11.4 & \\
\hline 329 & 562.4 & 5.67 & 0.217 & 0.258 & 50.7 & 16.3 & 0 & 7.8 & . \\
\hline 330 & 562.4 & 5.83 & 0.237 & 0.282 & 48.5 & 16.0 & 0 & 7.1 & \\
\hline
\end{tabular}


Table 2.5. Results of RTD and pressure drop experiments in a Kenics contactor with cocurrent upflow of air and coal slurry

Column Diameter $=2.54 \mathrm{~cm}$
Column Length $=160 \mathrm{~cm}$

\begin{tabular}{|c|c|c|c|c|c|c|c|c|}
\hline $\begin{array}{c}\text { Run } \\
\text { number }\end{array}$ & $\begin{array}{l}\text { Gas } \\
\text { flow } \\
\mathrm{cm}^{3} / \mathrm{se}=\end{array}$ & $\begin{array}{l}\text { Liquid } \\
\text { flow } \\
\mathrm{cm}^{3} / \mathrm{sec}\end{array}$ & $\begin{array}{l}\text { Liquid holdup, } \varepsilon_{L} \\
\text { (fraction of } \\
\text { total bed volume) }\end{array}$ & $\begin{array}{c}\text { Liquid saturation, } \\
\mathrm{R}_{\mathrm{L}} \text { (Fraction of } \\
\text { void volume) }\end{array}$ & $\begin{array}{c}\text { Peclet } \\
\text { number } \\
\text { Pe }\end{array}$ & $\begin{array}{l}\text { Dispersion } \\
\text { coefficient, } D_{L} \\
\mathrm{~cm}^{2} / \mathrm{sec}\end{array}$ & $\begin{array}{l}\text { Pressure } \\
\text { drop } \\
\Delta P \\
\text { in. } \mathrm{H}_{2} \mathrm{O}\end{array}$ & $\begin{array}{c}\text { Energy dissipation } \\
\text { per unit volume } \\
\frac{\mathrm{v}_{\mathrm{L}} \Delta \mathrm{P}_{\mathrm{f}}}{\mathrm{L}^{-3}} \\
\mathrm{ft} \cdot \mathrm{lb}_{\mathrm{f}} \cdot \mathrm{ft}^{-3} \cdot \mathrm{sec}^{-1}\end{array}$ \\
\hline 331 & 0 & 74.80 & 0.886 & 1.055 & 53.7 & 49.6 & 23.1 & 10.4 \\
\hline 332 & 0 & 77.50 & 0.971 & 1.156 & 50.6 & 49.8 & 22.7 & 6.0 \\
\hline 333 & 0 & 70.70 & 0.916 & 1.090 & 88.6 & 27.5 & 24.5 & 9.0 \\
\hline 334 & 98.1 & 79.80 & 0.660 & 0.786 & 71.0 & 53.8 & 9.8 & 19.3 \\
\hline 335 & 98.1 & 82.08 & 0.683 & 0.813 & 66.6 & 57.0 & 9.8 & 17.7 \\
\hline 336 & 134.7 & 81.17 & 0.621 & 0.739 & 105.6 & 39.1 & 9.8 & 23.6 \\
\hline 337 & 169.9 & 81.17 & 0.549 & 0.654 & 84.4 & 55.3 & $\begin{array}{r}14.0 \\
14.0\end{array}$ & 36.4 \\
\hline 338 & 204.4 & 79.80 & 0.469 & 0.558 & 78.2 & 68.7 & 17.5 & 53.1 \\
\hline 339 & 238.8 & 79.80 & 0.441 & 0.525 & 90.1 & 63.4 & 15.5 & 56.7 \\
\hline 340 & 268.3 & 77.52 & 0.411 & 0.489 & 56.3 & 105.9 & 15.4 & 62.2 \\
\hline 341 & 323.4 & 75.24 & 0.470 & 0.559 & 43.1 & 117.3 & 15.4 & 47.7 \\
\hline 342 & 378.3 & 70.68 & 0.381 & 0.453 & 49.1 & 119.3 & 15.4 & 64.2 \\
\hline 343 & 0 & 81.17 & 0.917 & 1.091 & 65.2 & 42.9 & 25.2 & 10.6 \\
\hline 344 & 137.1 & 80.71 & 0.659 & 0.785 & 86.1 & 44.9 & 16.0 & 24.5 \\
\hline 345 & 211.1 & 77.52 & 0.591 & 0.704 & 48.0 & 86.4 & 18.0 & 32.7 \\
\hline 346 & 276.4 & 77.52 & 0.562 & 0.668 & 115.7 & 37.7 & 20.0 & 38.3 \\
\hline 347 & 341.4 & 54.72 & 0.332 & 0.396 & 382.6 & 13.6 & 21.0 & 67.3 \\
\hline 348 & 397.7 & 77.52 & 0.502 & 0.597 & 147.9 & 33.0 & 30.0 & 57.8 \\
\hline 349 & 137.1 & 77.52 & 0.630 & 0.750 & 130.3 & 29.8 & 16.0 & 26.5 \\
\hline 350 & 207.8 & 77.52 & 0.564 & 0.672 & 53.9 & 80.5 & 17.0 & 35.4 \\
\hline 351 & 336.6 & 75.70 & 0.508 & 0.605 & 67.0 & 70.2 & 23.0 & 48.6 \\
\hline 352 & 0 & 76.61 & 0.846 & 1.007 & 70.1 & 40.8 & 30.0 & 17.1 \\
\hline 353 & 142.1 & 60.65 & 0.573 & 0.683 & 100.7 & 33.2 & 12.0 & 23.4 \\
\hline 354 & 218.3 & 58.37 & 0.513 & 0.611 & 49.4 & 72.7 & 38.0 & 47.8 \\
\hline 355 & 284.9 & 57.00 & 0.511 & 0.608 & 79.2 & 44.5 & 40.0 & 48.4 \\
\hline 356 & 356.6 & 58.37 & 0.460 & 0.548 & 813.9 & 4.9 & 41.0 & 59.3 \\
\hline
\end{tabular}


Table 2.5. (Continued)

\begin{tabular}{|c|c|c|c|c|c|c|c|c|}
\hline $\begin{array}{l}\text { Run } \\
\text { number }\end{array}$ & $\begin{array}{l}\text { Gas } \\
\text { flow } \\
\mathrm{cm}^{3} / \mathrm{sec}\end{array}$ & $\begin{array}{l}\text { Liquid } \\
\text { flow. } \\
\mathrm{cm}^{3} / \mathrm{sec}\end{array}$ & $\begin{array}{l}\text { Liquid holdup, } \varepsilon_{L} \\
\text { (fraction of } \\
\text { total bed volume) }\end{array}$ & $\begin{array}{c}\text { Liçuid saturation, } \\
\mathrm{R}_{\mathrm{L}} \text { (fraction of } \\
\text { void volume) }\end{array}$ & $\begin{array}{l}\text { Peclet } \\
\text { number } \\
\text { Pe }\end{array}$ & $\begin{array}{l}\text { Dispe-sion } \\
\text { coefficient, } D_{L} \\
\mathrm{sm}^{2} / \sec \end{array}$ & $\begin{array}{l}\text { Pressure } \\
\quad \text { drop } \\
\quad \Delta P \\
\text { in. } \mathrm{H}_{2} \mathrm{O}\end{array}$ & $\begin{array}{c}\text { Energy dissipation } \\
\text { per urit volume } \\
\frac{\mathrm{v}_{\mathrm{L}} \Delta \mathrm{P}_{\mathrm{f}}}{\mathrm{L}_{3}} \\
\mathrm{ft} \cdot \mathrm{lb}_{\mathrm{f}} \cdot \mathrm{ft}^{-1} \cdot \mathrm{sec}^{-1}\end{array}$ \\
\hline 357 & 414.0 & 54.72 & 0.416 & 0.495 & 250.0 & 16.6 & 50.0 & 72.2 \\
\hline 358 & 142.1 & 58.37 & 0.607 & 0.722 & 53.3 & 57.0 & 32.0 & 31.8 \\
\hline 359 & 0 & 58.37 & 0.879 & 1.046 & 75.2 & 27.9 & $\div 3.0$ & 16.9 \\
\hline 360 & 147.7 & 43.32 & 0.635 & 0.756 & 64.3 & 33.5 & 2.0 & 8.4 \\
\hline 361 & 222.0 & 40.74 & 0.563 & 0.670 & 92.6 & 24.7 & 3.0 & 12.2 \\
\hline 362 & 294.2 & 40.13 & 0.457 & 0.544 & 89.5 & 31.0 & 3.5 & 20.1 \\
\hline 363 & 356.3 & 38.00 & 0.413 & 0.491 & 67.2 & 43.3 & 4.0 & 23.5 \\
\hline 364 & 413.7 & 36.48 & 0.385 & 0.459 & 68.1 & 43.9 & 4.0 & 25.6 \\
\hline 365 & 119.3 & 97.58 & 0.674 & 0.803 & 152.2 & 30.0 & 25.0 & 36.2 \\
\hline 366 & 180.7 & 95.76 & 0.581 & 0.691 & 91.0 & 57.2 & 28.0 & 52.6 \\
\hline 367 & 227.0 & 89.38 & 0.462 & 0.550 & 46.0 & 132.7 & 38.0 & 86.5 \\
\hline 368 & 149.3 & 32.83 & 0.550 & 0.655 & 46.2 & 40.8 & 2.0 & 10.1 \\
\hline 369 & 233.1 & 31.31 & 0.485 & 0.577 & 58.9 & 34.6 & 3.0 & 13.6 \\
\hline 370 & 302.2 & $3 \overline{1} .62$ & 0.439 & 0.523 & 64.6 & 35.2 & 5.0 & 17.9 \\
\hline 371 & 534.8 & 31.01 & 0.377 & $0.449^{\circ}$ & 238.5 & 10.9 & 10.0 & 25.8 \\
\hline 372 & 397.4 & 29.79 & 0.327 & 0.389 & 95.9 & 30.0 & 15.0 & 33.9 \\
\hline 373 & 0 & 31.62 & 0.838 & 0.998 & 85.3 & 14.0 & 40.5 & 9.8 \\
\hline 374 & 157.7 & 25.23 & 0.569 & 0.677 & 48.1 & 29.1 & 0 & 6.5 \\
\hline 375 & 245.6 & 23.94 & 0.477 & 0.567 & 75.3 & 21.1 & 0 & 9.8 \\
\hline 376 & 326.3 & 24.17 & 0.425 & 0.506 & 64.3 & 27.9 & 0 & 12.7 \\
\hline 377 & 410.6 & 23.94 & 0.407 & 0.516 & 49.3 & 37.7 & 0 & 13.7 \\
\hline 378 & 479.0 & 23.48 & 0.377 & 0.449 & 94.4 & 20.8 & 0 & 15.5 \\
\hline 380 & 198.2 & 102.60 & 0.501 & 0.596 & 33.0 & 196.0 & 32.0 & 79.4 \\
\hline 381 & 254.2 & 113.00 & 0.634 & 0.755 & 20.6 & 273.3 & 36.0 & 60.9 \\
\hline 382 & 308.0 & 107.60 & 0.520 & 0.619 & 24.3 & 268.9 & 40.0 & 88.7 \\
\hline 383 & 357.6 & 114.00 & 0.493 & 0.586 & 23.9 & 306.4 & 42.0 & 105.5 \\
\hline 384 & 0 & 104.80 & 0.893 & 1.063 & 51.2 & 72.3 & 35.0 & 23.0 \\
\hline 385 & 0 & 22.50 & 0.776 & 0.924 & 100.3 & 9.1 & 12.0 & 3.2 \\
\hline 386 & 0 & 39.22 & 0.796 & 0.947 & 68.1 & $22 . .9$ & 16.0 & 6.2 \\
\hline 387 & 0 & 92.11 & 0.921 & 1.097 & 47.3 & 66.7 & 26.0 & 12.3 \\
\hline 388 & 331.7 & 86.64 & 0.509 & 0.606 & 31.4 & $171 . .4$ & 30.0 & 63.1 \\
\hline 389 & 331.7 & 88.96 & 0.500 & 0.595 & 62.2 & 90.4 & 30.0 & 66.8 \\
\hline 390 & 392.2 & 86.64 & 0.466 & 0.555 & 151.6 & 38.7 & 33.0 & 76.8 \\
\hline 391 & 362.0 & 59.30 & 0.485 & 0.578 & 28.5 & 135.5 & 12.0 & 32.8 \\
\hline
\end{tabular}


As noted in the preceding report, a suitable method for feeding solid $\mathrm{H}-\mathrm{COAL}$ vacuum still bottoms (VSB) to the carbonizer was being sought. The proposed method of slurrying the VSB with an organic liquid and pumping the slurry into the carbonizer proved unsuccessful. It has been possible to feed the VSB to the reactor in a semi-continuous manner pneumatically. Several small tubes are filled with VSB (or coal-VSB mixtures) before a run. When the reactor is up to temperature the VSB is impelled into the reactor with a blast of high pressure gas. Each tube contains about $15 \mathrm{~g}$ of feed, and tubes are fed at 5 minute intervals, so the feeding mechanism roughly simulates continuous operation. This feeding method has advantages over the slurry pumping method; since the VSB is not diluted with the solvent, analysis of products is simplified and the heat load in the reactor is reduced. In addition, a shorter vapor residence time is achieved.

To date one run has been completed. Table 2.6 summarizes the run conditions. Although analytical results are not yet available, it is possible to make some qualitative observations. The VSB formed some very hard agglomerates in the reactor. The size and shape of the agglomerates indicate that one or more liquid slugs were in the fluidized bed at the end of the run. The recovery of liquid products was disappointing; no materials fluid at room temperature were found in the condenser or condensate receiver. However, a very heavy tar did accumulate on the condenser tubing. Although recovery of this material was impossible, it appears to account for $10-20 \mathrm{wt} \%$ of the feed material. As much of this material as possible was scraped of the condenser tubing and sent for analysis.

More experiments using VSB and VSB-coal mixtures will be completed during the next month.

Table 2.6. Summary of conditions for PRC-27

\begin{tabular}{ll}
\hline Temperature & v1100 $\mathrm{F}$ \\
Pressure & 415 psia \\
Feed & $\mathrm{H}-\mathrm{Coal}$ Vacuum Still Bottoms \\
Mass fed & $67 \mathrm{~g}$ \\
Fluidizing gas & Methane \\
\hline
\end{tabular}




\subsection{References for Section 2}

1. J. P. Nichols (ed.), Coal Technology Program Progress Report for Apri1, 1977, ORNL/TM-5932.

2. J. P. Nichols (ed.), Coal Technology Program Progress Report for May, 1977, ORNL/TM-5980.

3. J. P. Nichols (ed.), Coal Technology Program Progress Report for July, 1977, ORNL/TM-6038.

4. F. Barretto, et al., "Three-Phase Flow Characteristics of Cylindrical Vessels," ORNL/MIT-257 (in preparation). 


\title{
3. MATERIALS ENGINEERING
}

\author{
R. T. King
}

The materials engineering and associated technology reported here are in support of activities directed by the Materials and Power Generation Branch of the Division of Materials and Exploratory Research, ERDA, in the Division of Fossil Energy. Other related work not funded directly by this division of ERDA/FE is included also.

\section{Summary}

Procedures are being developed with the DATA TRAK heat treating facility to allow preparation of relatively large heat treated samples; $21 / 4 \mathrm{Cr}-1$ Mo steel samples are being heat treated.

We are currently planning work on CoCrAlY coatings for FY-1978. After some initial difficulties, we have apparently located a supplier for the CoCrAlY material. We are completing some ultrasonic work on ceramic and cermet coatings which will carry over to the metallic coatings.

Cladding development work with type 320 stainless steels has focused on the effect of weld wire diameter on deposit integrity. The use of Inconel 82 to form intermediate layers in cladding is now being studied.

Testing of heat exchanger tube materials in the Fluidyne fluidized bed test unit continues.

Failure analysis and prevention work has continued with emphasis on solvent refined coal plants. Examination of erosion-corrosion test coupons exposed to a simulated gasifier environment at the Solar Corporation was started.

\subsection{Pressure Vessel and Piping Materials}

\section{A. Canonico}

We are developing procedures that will permit us to use the DA'l'A 'I'RAK (heat treating apparatus for microstructure simulation) to obtain specimen blanks that are large enough to allow the machining of $0.394 \mathrm{~T}$ compact tension specimens (CTS). These specimen blanks which measure $2.54 \mathrm{~cm}$ by $2.54 \mathrm{~cm}$ ( 1 in. by 1 in.) are considerably larger than any that have been used in the DATA TRAK. We are currently heat treating 0.394T CT specimen blanks that represent the surface and $1 / 4$ thickness location in quenched and tempered 203 to $305 \mathrm{~mm}$ ( 8 to $10 \mathrm{in.}$ ) $21 / 4 \mathrm{Cr}-1$ Mo steel plate. 


\subsection{Inspection Techniques for Wear- and Process-Resistant Coatings}

R. W. McClung and G. W. Scott

\subsubsection{Review and Evaluation (G. W. Scott)}

The requirements for the coatings project for FY-1978 have been detailed for NDT staff members and we are working out plans for experimental work.

\subsubsection{Specimens and Standards (G. W. Scott)}

We experienced some difficulty locating a supplier for powdered CoCrAlY alloy suitable for plasma spraying. Through additional contacts with S. J. Dapkunas of ERDA/FE and F. S. Pettit of Pratt and Whitney, we have identified Alloy Metals, Inc., Troy, Michigan, as a supplier. We have been alerted to expect some difficulty in obtaining coatings with appropriate density and oxidation resistance. There is a sensitivity to element distribution, especially yttrium, so some characterization work to assure that locally-produced specimens are of industrial quality majy be required.

\subsubsection{Ultrasonics (G. W. Scott)}

We received new transducers for our thickness gage and are testing them on ceramic and cermet specimens. Results are not complete at this time.

We constructed two small double-wedge probes, according to designs published previously, ${ }^{1}$ which interrogate thin specimens using a 45 -degree shear pitch-catch technique. We are conducting experiments on specimens with stepped coating thicknesses, both ceramic and cermet, and on specimens with unbond or missing-coating flaws. These experiments will show the feasibility of the method and provide a terminus for the ceramic/cermet work.

\subsection{Fossil Energy Welding and Cladding Program \\ D. P. Edmonds, J. D. Hudson, and J. J. Woodhouse}

During this reporting period we have investigated effects of welding wire diameter on deposit characteristics of type 320 stainless steel clads.

${ }^{1}$ K. V. Cook, "Ultraonic Methods," Coal Technology Program Technical Progress Report, March 1977, ORNL/TM-5883, sect. 3.2.7. 
Previous work has been done with $1.5 \mathrm{~mm}$ (0.06 in.) diam wire for the gas metal-arc (GMA) deposits and $3.0 \mathrm{~mm}$ ( $0.12 \mathrm{in.}$ ) diam wire for submergedarc (SA) deposits. Gas metal-arc deposits have been made with $0.9 \mathrm{~mm}$ (0.035 in.) wire in an attempt to increase the current density and stabilize the spray transfer mode of welding. However, it was found that more stable spray transfer had been obtained previously with the $1.5 \mathrm{~mm}$ wire. Submerged-arc deposits were made with $4 \mathrm{~mm}$ (0.156 in.) diam wire using welding conditions similar to previous deposits made with $3 \mathrm{~mm}$ wire. These deposits were found to be considerably more sensitive to fissuring than the previous deposits. Chemical analyses of these deposits are currently being performed to determine what compositional differences may exist between them. In addition we have begun to evaluate the feasibility of using an intermediate clad layer. Gas metal-arc deposits of intermediate layers have been made using $1.5 \mathrm{~mm}$ diam Inconel 82 filler wire. Stable welding conditions and fissure-free deposits were obtained for this material using pure argon shielding gas.

\subsection{Fireside Corrosion}

T. G. Godfrey, R. H. Cooper, and J. H. DeVan

The atmospheric fluidized bed facility at Fluidyne Corporation has continued to operate satisfactorily during this month. The $1000 \mathrm{hr}$ test is scheduled for completion on October 10, 1977, at which time the erosion/corrosion tube specimens will be removed and returned to ORNL for detailed examination. An ORNL staff member will be present when the tubes are removed from the combustor.

\subsection{Failure Analysis and Prevention}

T. G. Godfrey and J. H. DeVan

\subsubsection{Solar* Eros1on/Curruslun Cunpuns}

Examination of 56 erosion/corrosion coupons from the nominally $980^{\circ} \mathrm{C} 1000-\mathrm{hr}$ test by Solar in a simulated gasifier environment has begun. The samples are alloys, coated alloys, and pure ceramics and were either impacted by fine coal char at $90^{\circ}$ or $15^{\circ}$ from the surface, or subjected only to the semi-stagnant environment.

Preliminary inspection reveals that erosion was probably not severe since, in most instances, the front face of the coupon can not be distinguished from the rear. The alloy and coated coupons appear to have a tenacious scale, as yet unidentified. Selected samples will be sectioned for metallographic examination, phase identification, and possibly microprobe analysis and scanning electron microscopy.

*Division of Internation Harvester Company. 
3.5.2 Surveillance Coupons from Wilsonville, Alabama, Solvent Refined

Several racks, bearing a total of 52 corrosion surveillance coupons were removed by the plant operations staff after approximately six months' exposure in the Wilsonville, Alabama, Solvent Refined Coal Plant. The samples were weighed, sectioned, reweighed, and returned to the plant for reinsertion. The examination of these samples will progress slowly due to funding concerns.

\subsubsection{On-Site Inspection at Wilsonville, Alabama Solvent Refined coal rlant (G.W. Scott and B. C. Leslie)}

A team consisting of a metallographer and an engineer visited the SRC plant at Wilsonville, Alabama to inspect portions of the plant. Cracking of a type 410 stainless steel overlay on the carbon steel T105 fractionating tower was observed near manways. One small surface crack was observed in overlay weld metal on the Tl02 vacuum column. Detailed work is to be scheduled later. Some thickness measurement calibration work was performed. 
4. ALKALI METAL VAPOR TOPPING CYCLES

R. S. Holcomb and G. Samuels

4.1 Gas-Fired Potassium Boiler

R. S. Holcomb, D. B. Lloyd, and R. H. Guyman

\subsubsection{Contract Objective}

Design, construction, and testing of a full-scale potassium boiler tube bundle and burner module on water and then potassium to determine the performance and operating characteristics.

\subsubsection{Status Summary}

1. The potassium system installation was completed this month. Installation work done this month included the following:

a. Wiring connections to the electric heaters and thermocouples were completed.

b. The installation of the air preheater was completed.

c. Installation of the thermal insulation on the system components and piping was completed.

d. The side panels were installed on the fill and drain tank enclosure.

2. The fill and drain tank was filled with potassium.

3. The checkout of the instruments and controls was nearly completed.

\subsubsection{Plans for Next Month}

The checkout of the instruments and controls will be completed. The system will be filled with argon and evacuated at least twice in preparation for initial filling with potassium. Preheat tests will be run on the fill and drain tank to check out the performance of the electric heaters. Initial filling of the boiler with potassium is planned for about October 12, and shakedown tests will begin after that.

\subsubsection{Problem Areas}

The bypassing of combustion air across the leaking seal represents a potential problem in that if the fraction of the incoming air lost by leakage increases significantly at high air flow rates, the target power level of $4 \times 10^{6} \mathrm{Btu} / \mathrm{hr}$ for the potassium test may not be reached. It is currently planned to operate to as high a power level as possible with the leaking seal for the potassium test, and it is expected that it will be possible to operate to a power level close to $4 \times 10^{6} \mathrm{Btu} / \mathrm{hr}$. 


\subsection{Coal-Fired Alkali Metal}

G. Samuels

\subsubsection{Contract Objective}

The objective of the Coal-Fired Alkali Metal Power System Design Study is to establish a reference design for a 200-MW(e) alkali metal vapor/steam Rankine cycle system employing a coal-fired atmospheric fluidized bed furnace and to prepare a preliminary design of a fluidized bed furnace. This will be accomplished by studying the relative merits of both potassium and cesium vapor cycle systems and making a comparison of these systems. The results will be analyzed and potassium or cesium will be chosen as the cycle fluid and one of the systems will be selected as the reference. The reference system will be used for the preparation of a preliminary design of a fluidized bed furnace-boiler.

\subsubsection{Status Summary}

The study was completed in September and a draft report describing the design was issued. Cesium was selected as the working fluid for the topping cycle. The fuel used was Illinois No. 6 coal, and limestone was used as the bed sorbent material. For the reference design, the furnace operated at atmospheric pressure and the cycle conditions for the power conversion systems were $1500^{\circ} \mathrm{F}(1089 \mathrm{~K})$ to $900^{\circ} \mathrm{F}(756 \mathrm{~K})$ for the topping cycle and $2400 \mathrm{psi}(16.5 \mathrm{MPa}) / 1000^{\circ} \mathrm{F}(811 \mathrm{~K}) / 1000^{\circ} \mathrm{F}(811 \mathrm{~K})$ to $1 \mathrm{l} / 2 \mathrm{in}$. $\mathrm{Hg}$ $(5079 \mathrm{~Pa})$ for the steam system. Several variations of the plant were briefly evaluated. These variations included using a supercritical steam system and using a pressurized furnace.

The principal conclusions of the study are as follows:

1. A satisfactory design of an atmospheric pressure fluidized bed furnace binary power plant was evolved. The concept uses a variation of the conventional binary cycle which permits utilizing the full potential of the alkali metal topping cycle.

2. The net plant efficiency (coal to busbar) of the reference system was $44.6 \%$.

3. The net plant efficiency of a larger system with a 3500 psi $(24.1 \mathrm{MPa}) / 1000^{\circ} \mathrm{F}(811 \mathrm{~K}) / 1000^{\circ} \mathrm{F}(811 \mathrm{~K})$ steam system was $46.8 \%$.

4. An intermediate pressure turbocharged system with a furnace pressure of $4 \mathrm{~atm}(0.4 \mathrm{MPa})$ would have many advantages in comparison to the atmospheric pressure system, including (1) a plant efficiency about one percentage point higher than the reference design, (2) reduced limestone requirement, and (3) potential capital cost saving. 
5. Although cost estimates were not a part of the design study, a comparison of the design of this study to that of the Energy Conversion Alternative Study (ECAS) indicates plant costs 20 to $25 \%$ less than that of the final ECAS design.

\section{COAL EQUIPMENT TEST PROGRAM}

J. M. Holmes and R. E. MacPherson

TRW Energy Systems completed their subcontract activity to develop a comprehensive plan for the Coal Equipment Test Program (CETP). The TRW final report was received (TRW 97137-E001-RCC-00) which proposes a test program divided into three phases:

- Phase I Planning and Analysis

- Phase II Design and Modification of Test Facilities

- Phase III Component Test and Evaluation

A proposal was received from TRW to perform Phase I over a 9-month period. Funding has become available to proceed with this program and subcontract negotiations with TRW are currently in progress.

As a result of two recent meetings between ORNL, TRW, and Illinois Coal Gasification Group. (ICGG) representatives, negotiations for an agreement to obtain proprietary information concerning the ICGG process are currently in progress. In addition to the ICGG demo plant, another demo plant has been tentatively selected for evaluation of critical components by TRW. This demo is the medium-Btu Fuel Gas Plant recently announced by Memphis Light, Gas and Water Division, Memphis, Tennessee, and negotiations for a secrecy agreement with them will be started in October.

ORNL was requested by DOE/FE-MFPM to develop a program for testing coal feeders under development hy MFPM. A meeting was held at ORNL with Jet Propulsion Laboratory (JPL) personnel who have been coordinating this activity for MFPM. Agreement was reached that JPL will prepare the criteria for the feeder test program. The criteria will include:

- Type of feeders to be considered.

- Recommended operating conditions.

- Recommended coal throughput and run time.

- Recomnended feeder size and geometry.

Upon receipt of these criteria at the end of October, ORNL will proceed with the selection of a site and prepare a preliminary plan for a feeder test program. 


\section{FLUIDIZED BED COMBUSTOR TECHNOLOGY TEST UNIT}

R. S. Hölcomb

Preparation of the performance specifications for the combustor continued. The specification for the conceptual design was completed. It was reviewed internally and has been revised. The specification for the detailed design phase was completed and is now being reviewed. It is expected that the revision will be completed by about October 10 .

The final draft of the request for a Memorandum Directive was prepared and submitted for approval. A rough draft of the preliminary proposal for the formal project directive was completed except for the portion of the new cost estimate covering the conventional system components.

The possibility of installing the TTU system in Building 9731 rather than 9401-1 is being examined. The estimated cost for equipment removal and building modification is less for Building 9731 than for 9401-1. The possible rerouting of security fencing to allow visitors easier access is under study.

Several reports on industrial energy requirements have been reviewed as a part of the assessment study. These include reports covering a fairly broad range of industries and a few reports on particular industries. Most of the study on energy needs for a particular industry has been devoted to the petroleum, food, and paper industries. The petroleum industry has been identified from the work thus far as having energy needs in many processes that are well suited to being met by gas turbine cogeneration systems. Study of the food and paper industries is continuing and the study of several other industries will be initiated next month.

The corrosion test at Fluidyne was resumed on August 30. The furnace operated without incident during this month and the total running time at test conditions at the end of this month was about $730 \mathrm{hr}$. If no further interruptions occur, the 1000-hr test should be completed on October 12 . 
7. ENGINEERTNG STUDIES AND TECHNICAL SUPPORT

J. R. McWherter

\subsection{Process Modeling}

R. Salmon and D. M. Lister

\subsubsection{Contract Objective}

The objective is to assist the Fossil Energy (FE) Major Facility Project Management Division of DOE in its plan for computer analysis and computer support for coal conversion studies. This includes assistance to Purdue and Lehigh Universities in the development of computer programs for this plan.

\subsubsection{Status Summary}

I. Work continued on the heat exchanger design and cost estimation program HDC. Several numerical examples were mun using the Icarus Corporation COST code for comparison. The results were also checked against the costs presented by Fanaritis and Bevevino of Struthers Wells (Chemical Engineering, July 1976). Agreement of costs was very good in most cases.

2. The Purdue physical properties and material balance programs have been processed from the magnetic tape supplied by Purdue to a disk file. The disk file has been copied into a partitioned data set to facilitate separating the source and data files. The code has been processed through the EDITER program to obtain a numbered listing. Work is in progress using the EDITER program to revise the CDC coding as necessary for compatibility with the IBM system.

3. The ORNL cost estimating program CAPITAL was copied and sent to Purdue. This program uses the factor method to estimate the capital investment for complete process facilities based on major process equipment costs.

\subsubsection{Problem Areas}

Acquisition of process data on the ICGG gasification system remains a problem. The data are needed by Purdue and Lehigh in connection with the development of their simulation models, which are to be used on the ICGG flowshect. 


\subsection{Synthetic Fuels Process Research Digest}

R. W. Glass

\subsubsection{Contract Objective}

The objective is to provide continuing technical assistance to the Assistant Director for Processes of the Division of Materials and Exploratory Research (DMER) by preparing digest reviews of current or potential subjects relating to coal conversion technology.

\subsubsection{Status Summary}

1. Final review copies of "Flash Hydropyrolysis of Coal" and "Zinc Chloride Hydrocracking of Coal and Coal Extracts" were forwarded to contractors and DOE/FE. Comments were requested for an October 15 printing date. All articles for the first issue of the Digest have now reached the final review stage; no substantive changes are expected.

2. Four articles for the second issue of the Digest were previously indicated by $\mathrm{DOE} / \mathrm{FE}$. The four articles deal with (I) Consol's CSF process, (2) SRC-II, (3) Fischer-Tropsch, and (4) conversion of coal to low-Btu gas. The first article has been written and submitted for internal editorial review; the second manuscript is being written, and the last two subjects are being researched.

\subsection{Survey of Industrial Coal. Conversion Equipment Capabilities}

R. W. Glass and J. M. Holnes

\subsubsection{Contract Objective}

The general objective of this project is to conduct surveys of industrial equipment capabilities that will identify the present capability of industry to supply the equipment needed. The project will also determine research and development needs, including lead time requirements, for producing equipment of advanced design for the various unit operations of critical importance to the Major Facility Project Management Division's (MFPM) programs.

\subsubsection{Rotating Components (J. R. Horton, M. Siman-Tov, and W. R. Williams)}

1. The draft of the final report was completed and sent to T. K. Lau of DOE/FE-MFPM on october 3, 1977. The outline of that report is included as Table 7.1 .

2. A "library" of manufacturers' catalogs has been assembled for delivery to T. K. Lau at a later date.

3. It has been concluded that equipment should be available for nearly all expected clean stream applications likely to be found in coal conversion 
Table 7.1. Outline of the Final Report

1. INTRODUCTION

2. SUMMARY

3. DATA COLLECTION

3.1 Basis for Equipment Specifications

3.2 Equipment Characteristics

3.3 Questionnaires

3.4 Manufacturers

4. SURVEY RESULTS

4. 1 Pumps

4.2 Compressors

4.3 Hydraulic Turbines

4. 4 Gas Expanders

5. ANALYSIS AND DISCUSSION

5.1 Pumps

5.2 Compressors

5.3 Hydraulic Turbines

5.4 Gas Expanders

6. CONCLUSIONS AND RECOMMENDATIONS

G.I Availability

6.2 Research and Development Needs

6.3 Additional Areas of Study

References

Appendix A. PROCESSES FORMING A BASIS FOR EQUIPMENT SPECIFICATIONS

Appendix B. QUTETIONNAIRED

Appendix C. MANUFACTURERS RESPONDING TO ROTATING EQUIPMENT QUESTIONNAIRES

Appendix D. MANUFACTURERS' RESPONSES TO QUESTIONS ON AVAILABILITY AND CAPACITY 
facilities with the exception of oxygen compressors. Equipment which is critical to the development of the coal conversion industry and which needs to be developed or very significantly upgraded include slurry pumps and oxygen compressors as well as low pressure fans and blowers capable of handling dirty streams. Expanders, which will also require development, are not as critical although several processes will not be practical without them. Hydraulic turbines may be desirable for power recovery from slurry streams; however, their unavailability will not seriously affect any processes.

4. A presentation of the results of this survey will be made to T. K. Lau and several other MFPM personnel during the latter part of October. Subsequent to this, another presentation will be made in Washington for DOE personnel and others who may be invited.

7.3.3 Valves and Other Letdown Devices (W. A. Bush and E. C. Slade)

1. Preparation of the final draft report was completed.

2. An abstract was submitted of a presentation to be made at the "Workshop on Valves for Solids Handling Service on Coal Conversion" which is scheduled to be held in Morgantown, West Virginia, on November 14-16, 1977. Our presentation will summarize the results of the survey of the industrial capabilities of valve manufacturers.

7.3.4 Hot Gas Cleanup Devices (J. P. Meyer and M. S. Edwards)

During the reporting interval a draft of the final report has been completed. Comments received from external reviewers have been incorporated into the work.

\subsubsection{Heat Recovery Equipment Survey (W. R. Gambill and W. R. Reed)}

1. On September 1, the survey status was described during a meeting in Oak Ridge with the MFPM project manager. A summary was presented of the results available at that time.

2. Initial categorization of vendor literature in tabular form was completed.

3. Additional responses to the heat-exchanger questionnaire were received and a summary of all the responses was prepared.

4. Preparation of the draft report was essentially completed.

\subsection{Engineering Assistance}

J. M. Holmes

\subsubsection{Contract Objective}

The objective is to provide engineering assistance to the Fossil Energy Major Facilities Project Management Division of DOE. 
7.4.2 Status Summary

The assistance requested to date on this project has been provided and the total costs are now about equal to the budget. Therefore, no further assignments on this project are expected in FY 1977.

\subsection{Flash Hydropyrolysis}

$$
\text { S. P. N. Singh }
$$

\subsubsection{Contract Objective}

The objectives are to perform a scoping study on two conceptual flash hydropyrolysis processes and to determine if a more detailed engineering evaluation of one of these processes is justified.

\subsubsection{Status Summary}

1. Three copies of the draft of the final report on the study were sent to DOE/FE-MFPM on September 23, 1977, for their review and comments.

2. The work on the scoping study is essentially complete. Further work on the study, in terms of addressing the comments received from DOE/FE-MFPM, will be handled upon receipt of comments from DOE/FE-MFPM.

\subsection{Hot Gas Purification Processes \\ M. S. Edwards and J. P. Meyer}

\subsubsection{Contract Objective}

The objective of this project is to investigate the present state-ofthe-art hot gas cleanup processes. The application of most interest is the removal of contaminants (i.e., particulates, sulfurous gases, alkali metals, etc.) from coal-derived fuel gas prior to firing combined cycle turbines.

\subsubsection{Status Summary}

1. A number of concepts exist for high-temperature $\mathrm{H}_{2} \mathrm{~S}$ removal. Requests for information sent to vendors, $A-E$ firms, and others resulted. in the list shown in Table 7.2.

2. Although 16 processes (or concepts) are identified in Table 7.2, only eight are suitable for further consideration in this study. Two concepts are eliminated because they are proprietary and sufficient data have not been published; Battelle-Columbus solid sorption, and IGT/ Meissner molten metal concepts. Two are eliminated because they are proposed for $\mathrm{SO}_{2}$ rather than $\mathrm{H}_{2} \mathrm{~S}$ control; Hanna Mining iron oxide, and Shell oil copper oxide processes. Three of the listed processes are actually gasifiers, and control $\mathrm{H}_{2} \mathrm{~S}$ as an integral part of the gasification process; Westinghouse limestone gasifier, Kellogg molten-salt 
Table 7.2. High temperature $\mathrm{H}_{2} \mathrm{~S}$ removal processes

I. Solid sorption

A. Iron oxide

1. BuMines-Morgantown Energy Research Center (fixed bed)

2. Appleby-Frodingham (fluid bed)

3. Babcock \& Wilcox (iron plates)

4. University of Kentucky (coal ash)

5. Hanna Mining (flluid bed)

B. Proprietary: Battclle-Columbus

C. Dolomite/Limestone

1. Conoco Coal Development Co. (half calcined fluid bed)

2. Air Products (calcined fixed bed)

3. CCNY (half calcined panel bed)

4. Westinghouse (gasify with limestonne)

D. Copper oxide

1. Kennecott Copper (fluid bed)

2. Shell Oil (UOP licensor)

II. Liquid sorption

A. Molten salt

1. Battelle-NW (venturi scrub)

2. Kellogg (molten-salt gasifier)

3. Atomics International (molten-salt gasifier)

B. Molten metal-IGT/Meissner 
gasifier, and Atomics International molten-salt gasifier. Because these are not $\mathrm{H}_{2} \mathrm{~S}$ control processes, but rather gas producing processes, they are not considered further. Finally, the Air Products and Chemicals work on use of limestone led to their conclusion that limestone was not suitable for $\mathrm{H}_{2} \mathrm{~S}$ control.

3. Processes which will be evaluated for $\mathrm{H}_{2} \mathrm{~S}$ removal at high temperature are shown in Table 7.3 .

4. Data have been received from Acurex Aerotherm Corporation as to the particle size distribution of elutriated PFBC effluent obtained at the Exxon Miniplant in Linden, New Jersey.

5. Several novel concepts in high temperature-high pressure particulate cleanup have been identified. These include:

- Sonic augmentation to enhance particle agglomeration prior to collection.

- Dry scrubbing of raw product gas by using a venturi scrubber with recycled char as the scrubbing medium.

- High temperature-high pressure particulate removal via particle entrainment in coal slag based glasses.

Prior experience has shown that the first two collection techniques have been unable to either enhance or effect particulate removal in high temperature-high pressure applications. In the latter case, it appears that the utilization of molten salts will generate substantial problems in alkali carryover which will more than offset any advantages obtained for particulate control.

\subsubsection{Open Items}

1. We are still awaiting clarification as to specific references for proposed cleanup alternatives (e.g., cleanup technique used by USIC in their process for partial oxidation of naphthalene, and cleanup techniques downstream of ethylene cracking furnaces) suggested by MFPM.

\subsection{Large Oxygen Flant Study}

W. R. Reed and W. R. Gambill

\subsubsection{Contract Objective}

The objective of this study is to determine if significant economies of scale would be provided by building single-train cryogenic air separation plants with capacities in the range of 2000 to 20,000 TPD oxygen production. The oxygen purity is to be 95 vol. \% or higher; and the delivery pressure, within the range 1 to 70 atm abs., is to be determined by economics. 
Table 7.3. $\mathrm{H}_{2} \mathrm{~S}$ removal processes to be evaluated

I. Solid sorption

A. Iron oxide

1. BuMinco-MIERC

2. Appleby-Frodingham

3. Babcock \& Wilcox

4. University of Kentucky

B. Dolomite

1. Conoco Coal Development Co.

2. CCNY

C. Copper oxide: Kennecott Copper

II. Liquid sorption: Battelle NW molten salt 


\subsubsection{Status Summary}

our effort on this assignment was completed on September 9, when the summary report was transmitted to $D$. Garrett of DOE/F'-MFPM.

\subsection{State-of-the-Art Review of Heat Recovery Processes}

W. R. Gambill

\subsubsection{Contract Objective}

The objective of this review is to survey, appraise, and catalog the processes for heat recovery which industry offers or plans to offer or may logically be expected to offer. These processes will be applicable to the recovery of heat in process streams from primary exothermic coal conversion and combustion process steps. Simple unaugmented application of conventional heat transfer equipment will not be included. The review will distinguish between presently installable technology, near term developable technology, and future prospects. Consideration will be given to possible transfer of technology from other industries.

\subsubsection{Status Summary}

Work continued in the acquisition of additional literature and other information.

\subsection{Assessment of a Moving Bed System for Cleanup of Raw Gasifier Gas}

R. W. Glass, W. R. Gambill, and R. H. Lamb*

\subsubsection{Contract Objective}

The objective is to evaluate the feasibility of a proposed moving bed system for recovery of heat and removal of sulfur and particulates from raw gasifier gas.

\subsubsection{Status Summary}

1. A draft of the evaluation report for the subject system prepared by R. H. Lamb was received and reviewed at ORNL on September 23, 1977. Revisions were incorporated and two copies of the report returned to ORNL for subsequent use.

2. Discussions with Dr. T. Simpson (ERDA/FE-MFPM) were held at ORNL on September 30 to discuss the work effort. Final efforts for the subject study are pending MFPM review of the draft report. 
7.10 Technical/Economic Assessment of Hydrogen Production by the Steam/Molten Iron Process

R. W. Glass and L. Seglin*

\subsubsection{Contract Objective}

The objective is to determine if there is sufficient incentive to develop the subject process for production of hydrogen from coal.

\subsection{0 .2 Status Summary}

1. Pertinent information for the project has been gathered (Task I); kinetic data were not obtained, but these appear to be noncritical at this time.

2. Schematic flow sheets for three potential systems were prepared (Task 2); material balances (for the reactor sections) and energy efficiencies for the system were developed.

3. The three systems considered in part 2 are described as follows :

a. System 1 includes an oxidizer, a reducer, and a circulating slag heater. Product streams are $\mathrm{H}_{2}$ (100\%) and $\mathrm{CO}(100 \%)$. Cold gas efficiencies are $60 \%$ (no heat losses) and $52.2 \%$ (including heat losses). Carbon is used as the reductant.

b. System 2 includes a char gasifier, an oxidizer, a reducer, a circulating slag heater, and a $\mathrm{CO}_{2}$ removal plant. Product streams are $\mathrm{H}_{2}(100 \%)$ and $\mathrm{CO}$ (100\%). Cold gas efficiencies are $11.2 \%$ assuming no heat losses; with heat Iosses (estimated 50,000 Btu/hr/ft ${ }^{2}$ ) the system may be inoperable. $C O$ is used as the reductant.

c. System 3 includes an oxidizer and a reducer. Product streams are $\mathrm{H}_{2}(100 \%)$ and $\mathrm{CO}\left(36 \%\right.$ in $\left.\mathrm{N}_{2}\right)$, or $\mathrm{H}_{2}(100 \%)$, depending on the use of $\mathrm{CO}$ as a by-product. Cold gas efficiencies in the case of $\mathrm{H}_{2}$ and $\mathrm{CO}$ products are $74.8 \%$ (with no heat losses) and $73.4 \%$ assuming $5 \%$ heat loss, Assuming $\mathrm{H}_{2}$ as the only product, gas efficiencies are estimated at $4.8 \%$ (no heat losses) and $3.3 \%$ with $5 \%$ heat loss. Neither case would be operable at the $50,000 \mathrm{Btu} / \mathrm{ft}^{2} / \mathrm{hr}$ flux.

4. Discussions were held September 30, 1977 with Dr. Ted Simpson (DOE/FE-MFFM) at ORNL to discuss the subject work and pass on Task 3 effort. Essentially system I noted above was adopted with some

*Consultant. 
modification for continued study per the established work plan. Costs, equipment specifications, material and energy balances will not proceed on the selected system.

\subsection{A Study of Effluent Control Technologies for Hydrocarbon and Carbon Monoxide Emissions \\ from Coal Conversion Plants}

\section{J. Fisher and G. Peterson}

\subsubsection{Contract Objective}

The objective of this project is to provide a technical and economic comparison of processes available for the control of gaseous hydrocarbon (HC) and carbon monoxide (CO) emissions. The Lurgi gasification process and the HCOAL liquefaction process will be considered as reference source systems, and comparisons of control technologies will consider incineration as the reference control system.

\subsubsection{Status Summary}

1. Proposed EPA standards, as of November 176. for the $\mathrm{CO}_{2}$ rich streams from the Rectisol plant in the Lurgi SNG process are:

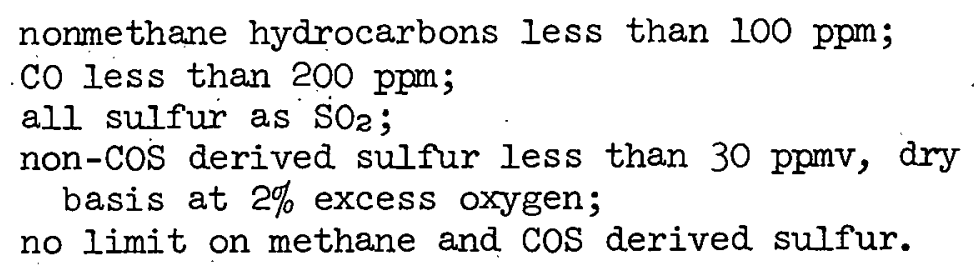

These regulations, when applied to processes other than incineration, require 99 plus \% removal of nonmethane hydrocarbons, 90 plus \% removal of $\mathrm{CO}$, and 99 plus \% removal of sulfur compounds.

2. The representative gas stream under investigation is:

\begin{tabular}{cc} 
Component & Moles $/ \mathrm{hr}$ \\
\cline { 2 - 2 } $\mathrm{CO}_{2}$ & $31,850 \mathrm{~mol} / \mathrm{h}$ \\
$\mathrm{H}_{2} \mathrm{~S}$ & 3 \\
$\mathrm{COS}$ & 3 \\
$\mathrm{C}_{2}{ }^{\prime} \mathrm{s}$ & 384 \\
$\mathrm{CO}$ & 65 \\
$\mathrm{CH}_{4}$ & 65 \\
$\mathrm{H}_{2}$ & $75 \mathrm{~mol} / \mathrm{h}$ \\
Temperature & $100^{\circ} \mathrm{F}$ \\
Pressure & $20 \mathrm{psia}$
\end{tabular}


The decontamination processes under investigation are:

Incineration

a. In a coal-fired boiler at $1800^{\circ} \mathrm{F}$.

b. In a medium-Btu gas-fired boiler at $1800^{\circ} \mathrm{F}$.

c. Over a catalyst bed at $700^{\circ}$ to $1000^{\circ} \mathrm{F}$.

Absorption

a. Decontamination by scrubbing with liquid $\mathrm{CO}_{2}$ or liquefaction of the $\mathrm{CO}_{2}$, followed by fractionation to produce a clean $\mathrm{CO}_{2}$ vent gas and a recycle thels stream.

b. $\mathrm{CO}_{2}$ absorption in hot carbonate or cold water.

Adsorption

On highly selective molecular sieves at low temperatures.

Diffusion

Through porous membranes.

3. No economic alternates to incineration have been found. While the accuracies of the costs of the alternate routes are in some cases poor, the cost differences between these routes and incineration are so large that the decision in favor of incineration is unquestionable.

The relative economics of the incineration alternates show catalytic oxidation to be somewhat cheaper than incineration in a coalfired boiler.

A sulfur, halide, and heavy metals resistant catalyst is claimed by Air Resources, Inc. The catalyst is reported to promote very little $\mathrm{SO}_{3}$ formation below $1100^{\circ} \mathrm{F}$. Its efficiency for ethane oxidation is in question. It has been commercially proven on mercaptans and halogenated hydrocarbons.

The cost of incineration in a coal-fired boiler remains uncertain. The ability of a boiler to handle the massive $\mathrm{CO}_{2}$ flows without loss of combustion efficiency must be resolved by a detailed study by a boiler manufacturer.

Adsorption as a separations route has been ruled out since no adsorbents which are sufficiently selective to economically separate $\mathrm{C}_{2} \mathrm{H}_{4}, \mathrm{CO}_{2}$, and $\mathrm{C}_{2} \mathrm{H}_{6}$ have been found.

Membrane separations costs have been estimated based on a projected membranes price from General Electric. However, membranes 
separations is still in the laboratory research stage. Projected costs show the approach to be non-competitive.

$\mathrm{CO}_{2}$ condensation and fractionation has proven much more expensive than originally estimated. Relative volatilities between the components are small. The $\mathrm{CO}_{2}-\mathrm{C}_{2} \mathrm{H}_{6}$ system is a minimum boiling azeotrope. Experimental data on the $\mathrm{CO}_{2}-\mathrm{C}_{2} \mathrm{H}_{6}$ system is being sought.

\subsubsection{Changes}

1. The study has been extended to include a search for commercially proven catalysts for the oxidation of the contaminants, at low, less than $1200^{\circ} \mathrm{F}$, temperatures.

\subsubsection{Problem Areas}

1. Accurate comparisons between the alternate incineration routes will require engineering efforts beyond the scope of this study.

2. The efficiency of Air Resources catalytic incineration process for ethane oxidation must be determined experimentally.

\subsubsection{Supporting Data}

See Table 7.4 for current capital and energy related cost projections. 
Table 7.4 Decontamination of Rectisol off-gases from Lurgi SNG Process Streams $(3)+(7)$ about $33,000 \mathrm{~m}$ of $98 \% \mathrm{CO}_{2} / \mathrm{h}$

\begin{tabular}{|c|c|c|c|c|c|}
\hline Process & $\begin{array}{c}\text { Capital } \\
\text { Investment } \\
\$ \mathrm{MM} \\
\end{array}$ & $\begin{array}{c}\text { Fuel } \\
\text { Recycle }\end{array}$ & $\begin{array}{l}\text { Fuel use } \\
\$ \mathrm{MM} / \mathrm{yr}^{1}\end{array}$ & $\begin{array}{l}\text { Steam use } \\
\$ \mathrm{MM} / \mathrm{yr}^{2}\end{array}$ & Catalyst \\
\hline \multicolumn{6}{|l|}{ Incineration } \\
\hline Coal-fired boiler & 9 & 0 & $<0.27>$ & 20 & \\
\hline $\begin{array}{l}\text { Medium-Btu gas-fired boiler } \\
\text { with recuperator }\end{array}$ & 6 & 0 & 12 & $<5>$ & \\
\hline Catalytic oxidation & approx. 14 & 0 & 0 & $<1.8>$ & 0.3 \\
\hline \multicolumn{6}{|l|}{ Absorption } \\
\hline $\begin{array}{l}\text { Condensation of } \mathrm{CO}_{2} \text { and } \\
\text { fractionation }\end{array}$ & 100 & 8 & 0 & 13 & \\
\hline Hot carbonate scrubbing & 38 & 8 & 0 & 19 & \\
\hline Cold water scrubbing & 100 & 8 & 0 & 8 & \\
\hline \multicolumn{6}{|l|}{ Adsorption } \\
\hline \multicolumn{6}{|l|}{$\begin{array}{l}\text { No practical adsorbent } \\
\text { has been found }\end{array}$} \\
\hline \multicolumn{6}{|l|}{ Membrane Separations } \\
\hline $\begin{array}{l}\text { Ultra-thin silicone rubber } \\
\text { membranes }\end{array}$ & about 75 & 8 & 0 & 6 & . \\
\hline
\end{tabular}

$1_{\text {Coal }}$ is priced at $\$ 0.3 / \mathrm{MMBtu}$; medium-Btu gas is priced at $\$=.50 \mathrm{MMBtu}$.

${ }^{2} 900 \mathrm{Ib} / 825^{\circ} \mathrm{F}$ steam is $\$ 1.35 / \mathrm{M}$ Ib (assuming $30 \phi$ coal).

3 Recycle contaminated fuel gas is priced at $\$ 3 /$ MMBtu. 


\title{
8. PROCESS AND PROGRAM ANALYSIS
}

\author{
J. R. McWherter
}

Process and program analysis studies are being conducted for the DOE Fossil Energy Office of Program Planning and Analysis. This effort includes research studies on most of the coal conversion and utilization processes. The program objective is to provide, on a consistent basis, technical and. economic evaluations of competing processes and systems for coal conversion and utilization.

\subsection{Low Btu Coal Gasification}

H. F. Hartman, D. E. Reagan, and J. P. Belk

Activities in September 1977 involved work on completing the process data, process comparisons, and process considerations sections and preparation of the project draft report. The report concludes the study phase of this project. Additional work will be done to distribute the draft report, obtain comments, and prepare the final report. Comments will be requested from ORNL personnel on the complete draft and from process proponents on their specific process subsections.

\subsubsection{Process Data}

Technical data subsections were completed for the 21 processes that had been selected for detailed investigation. Processes were classified by gasifier type; namely, fixed (moving) bed, fluidized bed, entrained flow and molten bath. A single-page summary was followed by the topics noted: process status, description, operating conditions, reactants, utilities, products, efficiency, test results and problem areas. References were then listed for each process.

\subsubsection{Process Comparisons}

This section was completed and contained both generic and specific comparisons of coal gasification processes. Characteristics of coal gasifiers were compared by gasifier type and included experience, complexity, capacity, inventory, coal feed and handling, product gas, ash removal, temperature, and operating range. The characteristics were first described, then summarized and compared using $a+,-$, and 0 method of judging the relative merits of each characteristic. Processes described in the technical data subsections were compared for the following topics: status and location, gasifier arrangement, gasifier capacity, gasifier operating conditions, oxidant and steam requirements, efficiencies of coal to product gas, product gas composition, coal requirements, and coal feeding methods. A table was prepared for each topic. 


\subsubsection{Process Considerations}

This section was completed and contained a discussion of problem areas, economics and process applications. Problem areas included an overview of the following general problems associated with most gasifiers: coal types, coal handling, coal feeding, carbon conversion, thermal efficiency, product treatment, turndown/load following capability, complexity/scale-up, erosion and wear, and refractory materials. The economics section included gas cost and investment figures that have been estimated and reported by others for three systems; namely, small gasifiers, large gasifiers, and pipeline gas systems. The process applications section included a description of gas characteristics (low, medium, and high Btu), suggested process applications by gasifier type and operating pressure (atmospheric, pressurized) and suggested specific applications for the 21 processes investigated in detail. The suggested uses were for applications such as fuel gas (small, large scale), power generation (retrofit, combined cycle) and synthesis gas (chemicals, pipeline gas).

\subsubsection{Report Planning}

A draft report was prepared and represented work done in this study from July 1976 through September 1977. The draft report contains the following sections: abstract, introduction and summary, process summary, screening and selection, process data, process comparisons, and process considerations.

\subsubsection{Summary of Activities}

Work done on this project can be summarized as follows.

97 processes or gasifiers were surveyed.

59 processes (of the 97 ) were summarized.

21 processes were selected for more detailed study.

Process data were reviewed and summarized for the 21 .

Processes were compared generically and specifically.

Problem areas, economics and applications were discussed.

\subsubsection{Future Work}

This study phase of the project is complete. Future work pertains to the distribution of the draft report and preparation of the final report. The draft report will be distributed for ORNL review; comments will be obtained and considered in preparation of the final report. Drafts of specific process data subsections will also be distributed to the process proponents; their comments will be requested and considered for the final report. 


\subsection{Direct Combustion}

E. C. Fox, T. D. Anderson, and H. I. Bowers

\subsubsection{Objectives}

The purpose of this study program is to assist DOE/FE in their effort to develop a National strategy to increase the near-term use of coal through direct combustion; the applications of interest in this study are the small-to-moderate industrial user and the large residential/ commercial user. The following objectives will be accomplished:

1. Identify and quantify the important factors restricting the use of coal in the sectors of interest.

2. Evaluate potential technological and institutional solutions to the problems identified in (I) above.

3. Make recommendations to $\mathrm{DOE} / \mathrm{FE}$ relative to the most promising approaches to increasing the near-term use of coal.

\subsubsection{Results}

The economic analysis for on-site direct combustion for small-moderate industrial and commercial/residential users has been. completed. The results indicate that in comparison with firing oil under current conditions, small industrial users (less than 250,000 lb/hr steam use) have little economic incentive to shift to coal. For larger size, the results show promising incentives (especially with the new investment tax credits and boiler fuel taxes) for regions near coal fields and less exciting for users distant from the coal sources. For residential/commercial users, the incentives are less attractive. The low capacity factors inherent in space heating applications make a capital intensive system such as coal look unattractive in comparison to oil under present economic conditions.

The details of these results, along with other portions of the assessment, are being compiled into a draft report for $\mathrm{DOE}^{\prime} \mathrm{s}$ review.

\subsection{Advanced Power Conversion Systems}

All twelve reports covering this work were completed and sent to OPPA for review before September. No additional work was done in September on this project.

\section{4 Li quefaction}

The Ralph M. Parsons Company is working under subcontract on this subprogram with J. B. O'Hara as Project Manager. 


\subsubsection{Survey of Liquefaction Processes}

The preliminary report (Data Source Book) summarizing the characteristics of 32 liquefaction processes reviewed during the screening survey has been edited. Draft copies will be submitted to ORNL for review prior to final printing.

\subsubsection{Detailed Review of High Potential Liquefaction Processes}

As a result of the assessments, Parsons recommended selection of the following five processes for more detailed comparison:

1. H-Coal; a catalytic hydroliquefaction process

2. CSF; a donor solvent process

3. SRC; a non-catalytic/pseudocatalytic hydroliquefaction process

4. Fischer-Tropsch using flame-sprayed catalytic heat exchange service; an indirect process

5. Gasoline via Mobil-M; an indirect liquefaction process followed by catalytic conversion of methanol to gasoline

The recommended selection of a donor solvent process is based on the availability of information to parsons to properly evaluate the technology.

\subsection{High Btu Gas}

This subprogram is being analyzed under subcontract by the Scientific Design Company, Incorporated (SD) with A. S. West as Project Manager.

\subsubsection{Contract Objective}

The objective of the work is to provide technical and econonic evaluations of competing processes, concepts, and systems for the production of high Btu gas from coal.

\subsubsection{Status Summary}

'Ihe Battelle Agglomerating Ash Burner Process Evaluation for the production of high Btu gas has been finalized. A copy has been sent to Dr. L. M. Joseph for his review and approval prior to issue. Because of the delay in finalizing this report a two-month no cost increase extension of the subcontract has been requested. 


\title{
8.6 In Situ Coal Gasification
}

\author{
W. C. Ulrich
}

\subsection{Contract Objective}

The objective of this program is to provide technical and economic evaluations of candidate processes for the conversion of coal in situ to fluid products presently of interest.

\subsubsection{Status Summary}

1. Writing of the draft economic evaluation report, "Process Designs and Economic Evaluations for the Linked Vertical Well In Situ Coal Gasification Process," was completed. Copies were transmitted to OPPA for review as scheduled in the project work statement.

2. Editing of the report, "Evaluation of In Situ Coal Gasification on a Regional Basis," was completed and preparations for publication were initiated.

\subsubsection{Open Items}

Review by OPPA (and others) of the draft economic evaluation report, "Process Designs and Economic Evaluations for the Linked Vertical Well In Situ Coal Gasification Process," is continuing.

\subsection{Coal Beneficiation}

G. R. Peterson, S. P. N. Singh, and R. Salmon

\subsubsection{Contract Objective}

The objective of this project is to provide technical and economic evaluations of current beneficiation processes.

\subsubsection{Status Summary}

1. The economic analyses of the mechanical beneficiation and the Battelle Hydrothermal Coal processes have been completed for both the grassroots and the battery limits type plants. The above economic analyses were performed for plants processing Pennsylvania, Illinois, and Western Kentucky coals.

2. A computer program is being developed which will have the computer draw the plots which result from the economic analyses. The computer program has been written and is in the final "debugging" stage.

3. Economic analyses are presently being performed for conceptual plants based on the TRW-Meyers coal beneficiation process. 
4. The draft of the final report on the coal beneficiation study is being retyped to include the changes and additions requested by $\mathrm{DOE} / \mathrm{FE}-\mathrm{OPFA}$.

\subsection{Gas Cleanup Studies}

M. S. Edwards and R. Salmon

\subsubsection{Contract Objective}

The objective of the gas cleanup study is to collect information on the technology and economics of processes for the removal of impurities (principally hydrogen sult'ide) t'rom fuel gas streams. High and low temperature cleanup processes will be reviewed.

\subsubsection{Status Summary}

1. Process descriptions have been written for the Molecular Sieve and high-temperature Iron Oxide processes.

2. The vendors for the Sulfinol and Molecular Sieve processes and the developer of the high-temperature Iron oxide process have returned comments on their respective processes. Only minor corrections were required.

\subsubsection{Open Items}

1. Vendor responses have not yet been received for the MDEA and stretford processes. The first draft report will not be delayed because of this, but vendor comments will be incorporated into the final version published.

\subsection{The Potential of the Synthesis of Chemicals from Coal as a DOE Research Program}

This work is being done under subcontract by the Radian Corporation with D. N. Garner as the Project Director.

The final draft report was completed and submitted to ORIL and DOE/ FE-OPPA for review. 
9. FOSSIL ENERGY ENVIRONMENTAL PROJECT

C. R. Boston

\subsection{Landfill Storage of Coal Conversion} Solid Wastes/Information Assessment

H. M. Braunstein

Finalization of the document is proceeding with delivery to DOE expected in November 1977.

\subsection{Landfill Storage of Coal Conversion Solid Wastes/Experimental Phase}

W. J. Boegly, Jr.

Samples of solid waste slurry from the Hygas pilot plant have been received. No feed coal was received but IGT has been contacted and a 55-gal drum of this material should be in transit to ORNL. No word was received on the Battelle Agglomerating Burner waste during September.

A major effort was spent on preparing the annual report for FY 1977 and revising and detailing the program plan for FY 1978.

Staffing for this task is expected to be complete by October 1 .

\subsection{Environmental Monitoring Handbook}

S. G. DeCicco

The Handbook is now being used extensively by contractors and their environmental subcontractors in designing monitoring programs for the nation's first fossil demonstration plants (pipeline gas). This has become an extremely important position document in our role as advisors to the contractors in developing the environmental reports and ultimately in our role as authors of the environmental impact statements.

Discussions were held with Hershul Jones (DOE) and Radian Corporation on August 3 and again on August 11 regarding possible additions to the Operation part of the Handbook. It was agreed that Radian would contribute a subsection on process monitoring. This will be incorporated, along with other changes, in Revision 3 of the document. The intense involvement with contractor interaction will require a delay in delivery of Revision 3 to December 1, 1977. This will cause no delay in ultimate delivery of the completed document since the Radian contribution is also due on December 1, 1977. 


\title{
9.4 Programmatic Assessment/Pipeline Gas
}

\author{
S. G. DeCicco
}

This task has been placed on "hold" at DOE request (telcon, H. T. Jones, August 8, 1977).

\subsection{Programmatic Assessment/Fuel Gas}

S. G. DeCicco

Th1s task has been placed on "hold" at DOE requcst (telcon, H. T. Jones, August 8, 1977).

\subsection{Technical Assistance}

Considerable team effort went into this task during the month of September, mainly in interaction with contractors in preparing environmental monitoring programs. A number of meetings were held to discuss proposed monitoring plans. Fossil Energy Environmental Project members participated in a site visit to the CONOCO demo plant location and in a two-day work session at Westinghouse Environmental Systems Department (WESD), Pittsburgh, to assist the environmental subcontractor in developing a detailed monitoring program. 


\section{INTERNAL DISTRIBUTION}

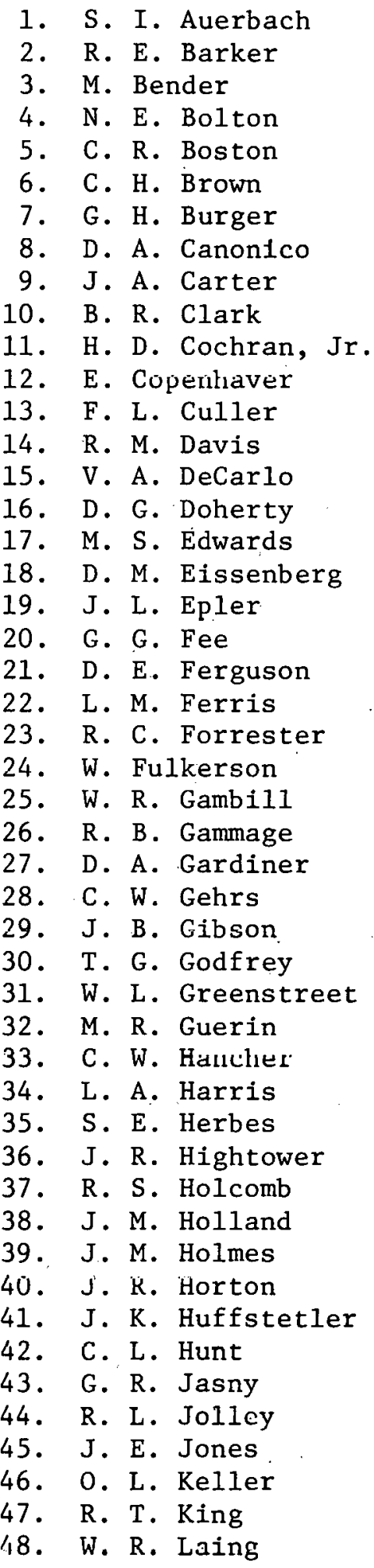

49. R. S. Livingston

50. R. E. MacPherson

51. A. P. Malinauskas

52. G. B. Marrow

53. C. J. McHargue

54. L. E. McNeese

55. J. R. McWherter

56. H. J. Metz

57. W. R. Mixon

58. J. E. Mrochek

59. P. Nettesheim

60-64. J. P. Nichols

65. B. Niemann

66. L. C. Oakes

67. G. E. Oswald

68. G. R. Peterson

69-70. T. W. Pickel

71. H. Postma

72. D. E. Reichle

73. C. R. Richmond

74. B. R. Rodgers

75. M. W. Rosenthal

76. R. H. Ross

77. T. H. Row

78. W. L. Russell

79. R. Salmon

80. G. Samuels

81. C. D. Scott

82. D. S. Shriner

83. W. D. Shults

84. S. P. N. Singh

85. C. B. Smith

86. G. P. Sulth

87. I. Spiewak

88. R. L. Spore

89. E. G. St. C1air

90. J. B. Storer

91. R. A. Strehlow

92. A. J. Thompson

93. D. B. 'lrauger

94. W. C. Ulrich

95. P. R. Vanstrum

96. J. S. Watson

97. J. R. Weir

98. P. R. Westmoreland

99. M. K. Wilkinson

100. L. V. Wilson

101. C. S. Yust 
102. Patent office

103. Lab. Records-RC

104-110. Lab. Records

111-113. Central Research Library

114. Document Reference Section

EXTERNAL DISTRIBUTION

DOE, Oak Ridge Operations

115. Research and Technical Support Division

DOE, San Francisco Operations

116. N. S. Hagen

DOE, Washington

117. W. Bakker, MER

138. T. K. Lau, MFPM

118. N. F. Barr, DTO

139. R. W. A. LeGassie, APA

119. J. D. Batchelor, CCU

140. J. L. Livermäl, AES

120. R. Beck, CCU

121. T. Beresovski, RDD

122. L. M. Burman, OGST

123. E. L. Clark, CCU

124. N. P. Cochran, MFPM

125. T. Cox, MER

126. P. Duhame1, MER

127. J. Forst, MER

128. H. Franke1, MER

129. S. I. Freedman, CCU

141. W. G. McDaniel, MFPM

130. D. Garrett, MFPM

142-147. C. Miller, CCU

148. G. A. Mills, MER

149. W. E. Mott, DBER

150. M. B. Neuworth, CCU

151. E. S. Pierce, DPR

152. H. E. Podal1, MFPM

153. J. L. Powe11, MFPM

154. J. W. Ramsey, OGST

155. M. Reilly, FE/OPPA

131. S. W. Gouse, AFE

156. John Shen, MER

157. A. P. Sikri, OGST

132. W. S. Harmon, MFPM

158. D. K. Stevens, DPR

133. R. M. Jimeson, DTO

159. J. W. Watkins, OGST

134. H. R. Johnson, FE/OPPA

160. P. C. White, AFE

135. H. Jones, MFPM

136. L. Kindley, MER

161. P. R. Wieber, OGST

137. C. Knudsen, FE/OPPA

162. R. W. Wood, DBER

Department of Housing and Urban Development, 4517 th Street, S.W., Washington, DC 20410

163. G. S. Leighton

164. J. H. Rothenberg

University of Kentucky, Institute for Mining and Materials Research, 213 Bradley Hall, Lexington, KY 40506

165. Theresa Wiley, Institute Librarian

166. 0. J. Haun

167. J. K. Shau

National Science Foundation, 1800 G Street, N.W., Washington, DC 20550

168. Robert Rabin 
169. Charles B. Sedman, U.S. Environmental Protection Agency, Research Triangle Park, NC 27711

170. N. S. Boodman, Section Supervisor, U.S. Steel Corporation Applied Research Laboratory, 125 Jamison Lane, Monroeville, PA 15146

171-182. ERDA Pittsburgh Energy Research Center, U.S. Energy Research and Development Administration, Attention: Director for J. P. Barreca, 4800 Forbes Ave., Pittsburgh, PA 15213

183-188. The Director, Morgantown Energy Research Center, P.0. Box 800, Morgantown, WV 26506

189. Tetra Tech, Inc., 1911 N. Ft. Myer Drive, Suite 601, Arlington, VA 22209, Attention: Walter McGough, Jr.

190. Cameron Engineers, Attn: Gary L. Baughman, 1315 South Clarkson St., Denver, CO 80213

191. Y. A. Liu, Department of Chemica1 Engineering, Auburn University, Auburn, Alabama 36830

192. W. E. Warnke, Coal Preparation Research Manager, U.S. Bureau of Mines, 2401 E Street N.W., Washington, DC 20241

193-218. Technical Information Center, ERDA 